\title{
GAMMA-RAY AND RADIO PROPERTIES OF SIX PULSARS DETECTED BY THE FERMI LARGE AREA TELESCOPE
}

P. Weltevrede ${ }^{1,2}$, A. A. Abdo ${ }^{3,4}$, M. AcKermanN ${ }^{5}$, M. Ajello ${ }^{5}$, M. Axelsson ${ }^{6,7}$, L. Baldini $^{8}$, J. Ballet ${ }^{9}$, G. Barbiellini ${ }^{10,11}$, D. Bastieri ${ }^{12,13}$, B. M. Baughman ${ }^{14}$, K. BeChtol ${ }^{5}$, R. Bellazzini ${ }^{8}$, B. Berenit ${ }^{5}$, E. D. BloOM ${ }^{5}$, E. Bonamente ${ }^{15,16}$, A. W. Borgland ${ }^{5}$, J. Bregeon ${ }^{8}$, A. BreZ ${ }^{8}$, M. Brigida ${ }^{17,18}$, P. Bruel ${ }^{19}$, T. H. Burnett ${ }^{20}$, S. Buson $^{13}$, G. A. Caliandro ${ }^{17,18}$, R. A. Cameron ${ }^{5}$, F. Camilo ${ }^{21}$, P. A. Caraver ${ }^{22}$, J. M. Casandjian ${ }^{9}$, C. Cecchi ${ }^{15,16}$, Ö. Çelik ${ }^{23,24,25}$, E. Charles ${ }^{5}$, A. Chekhtman ${ }^{3,26}$, C. C. Cheung ${ }^{3,4,23}$, J. Chiang ${ }^{5}$, S. Ciprini ${ }^{15,16}$, R. Claus ${ }^{5}$, I. Cognard ${ }^{27}$, J. Cohen-Tanugi ${ }^{28}$, L. R. Cominsky ${ }^{29}$, J. Conrad ${ }^{7,30,64}$, S. Cutini ${ }^{31}$, C. D. Dermer ${ }^{3}$, G. Desvignes ${ }^{27}$, A. De Angelis ${ }^{32}$, A. DE LuCA ${ }^{22,33}$, F. De Palma ${ }^{17,18}$, S. W. Digel ${ }^{5}$, M. Dormody ${ }^{34}$, E. Do Couto e Silva ${ }^{5}$, P. S. Drell ${ }^{5}$, R. Dubois ${ }^{5}$, D. Dumora ${ }^{35,36}$, C. Farnier ${ }^{28}$, C. Favuzzi $^{17,18}$, S. J. Fegan ${ }^{19}$, W. B. Focke ${ }^{5}$, P. Fortin ${ }^{19}$, M. Frailis ${ }^{32}$, P. C. C. Freire ${ }^{37}$, P. Fusco ${ }^{17,18}$, F. Gargano ${ }^{18}$, D. Gasparrini ${ }^{31}$, N. Gehrels ${ }^{23,38}$, S. Germani ${ }^{15,16}$, G. Giavitto $^{39}$, B. Giebels ${ }^{19}$, N. Giglietto ${ }^{17,18}$, F. Giordano ${ }^{17,18}$, T. Glanzman ${ }^{5}$, G. Godfrey ${ }^{5}$, I. A. Grenier ${ }^{9}$, M.-H. Grondin ${ }^{35,36}$, J. E. Grove ${ }^{3}$,

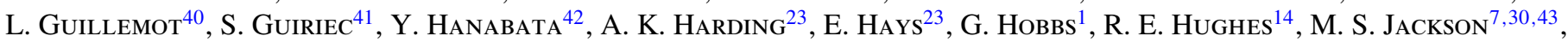

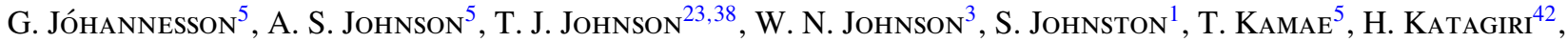
J. Kataoka ${ }^{44,45}$, N. Kawai ${ }^{44,46}$, M. Keith ${ }^{1}$, M. Kerr ${ }^{20}$, J. KNÖDlseder ${ }^{47}$, M. L. Kocian ${ }^{5}$, M. Kramer ${ }^{2,40}$, M. Kuss ${ }^{8}$, J. Lande ${ }^{5}$, L. LATronico ${ }^{8}$, M. Lemoine-Goumard ${ }^{35,36}$, F. Longo ${ }^{10,11}$, F. LoparCo ${ }^{17,18}$, B. LotT ${ }^{35,36}$, M. N. Lovellette ${ }^{3}$, P. Lubrano $^{15,16}$,

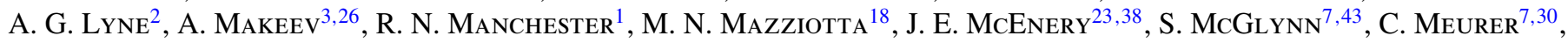

P. F. Michelson ${ }^{5}$, W. Mitthumsiri ${ }^{5}$, T. Mizuno ${ }^{42}$, A. A. Moiseev ${ }^{24,38}$, C. Monte ${ }^{17,18}$, M. E. MonZani ${ }^{5}$, A. Morsellit ${ }^{48}$, I. V. Moskalenko ${ }^{5}$, S. Murgia ${ }^{5}$, P. L. Nolan ${ }^{5}$, J. P. Norris ${ }^{49}$, E. Nuss ${ }^{28}$, T. Ohsugi ${ }^{42}$, N. OMOdei ${ }^{8}$, E. Orlando ${ }^{50}$, J. F. Ormes ${ }^{49}$, D. Paneque ${ }^{5}$, J. H. Panetta ${ }^{5}$, D. Parent ${ }^{35,36}$, V. Pelassa ${ }^{28}$, M. PePe ${ }^{15,16}$, M. Pesce-Rollins ${ }^{8}$, F. Piron ${ }^{28}$, T. A. Porter ${ }^{34}$, S. Rainò ${ }^{17,18}$, R. Rando ${ }^{12,13}$, S. M. Ransom ${ }^{51}$, M. Razzano ${ }^{8}$, N. Rea ${ }^{52,53}$, A. Reimer ${ }^{5,54}$, O. Reimer ${ }^{5,54}$, T. RePoseur ${ }^{35,36}$, L. S. Rochester ${ }^{5}$, A. Y. Rodriguez ${ }^{52}$, R. W. RoMANI ${ }^{5}$, M. Roth ${ }^{20}$, F. RYDE ${ }^{7,43}$, H. F.-W. SADROZINSKI ${ }^{34}$,

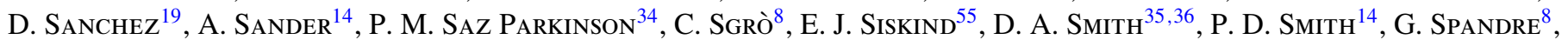
P. Spineldi ${ }^{17,18}$, B. W. Stappers ${ }^{2}$, M. S. Strickman ${ }^{3}$, D. J. Suson ${ }^{56}$, H. TAJima ${ }^{5}$, H. TAKahashi ${ }^{42}$, T. TAnaka ${ }^{5}$, J. B. ThaYer ${ }^{5}$,

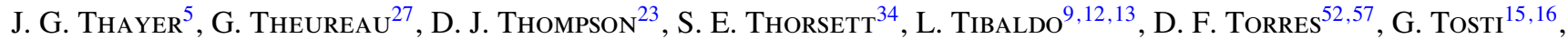

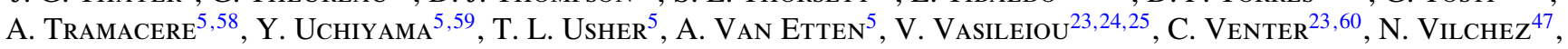

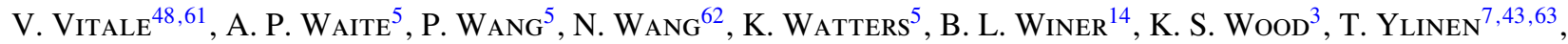
AND M. ZIEGLER ${ }^{34}$

${ }^{1}$ Australia Telescope National Facility, CSIRO, Epping NSW 1710, Australia; patrick. weltevrede@ manchester.ac.uk, Simon.Johnston@atnf.csiro.au

${ }^{2}$ Jodrell Bank Centre for Astrophysics, School of Physics and Astronomy, The University of Manchester, M13 9PL, UK

${ }^{3}$ Space Science Division, Naval Research Laboratory, Washington, DC 20375, USA

${ }^{4}$ National Research Council Research Associate, National Academy of Sciences, Washington, DC 20001, USA

${ }^{5}$ W. W. Hansen Experimental Physics Laboratory, Kavli Institute for Particle Astrophysics and Cosmology, Department of Physics and SLAC National Accelerator Laboratory, Stanford University, Stanford, CA 94305, USA

${ }^{6}$ Department of Astronomy, Stockholm University, SE-106 91 Stockholm, Sweden

7 The Oskar Klein Centre for Cosmoparticle Physics, AlbaNova, SE-106 91 Stockholm, Sweden ${ }^{8}$ Istituto Nazionale di Fisica Nucleare, Sezione di Pisa, I-56127 Pisa, Italy

${ }^{9}$ Laboratoire AIM, CEA-IRFU/CNRS/Université Paris Diderot, Service d'Astrophysique, CEA Saclay, 91191 Gif sur Yvette, France

${ }^{10}$ Istituto Nazionale di Fisica Nucleare, Sezione di Trieste, I-34127 Trieste, Italy ${ }^{11}$ Dipartimento di Fisica, Università di Trieste, I-34127 Trieste, Italy

12 Istituto Nazionale di Fisica Nucleare, Sezione di Padova, I-35131 Padova, Italy

${ }^{13}$ Dipartimento di Fisica "G. Galilei," Università di Padova, I-35131 Padova, Italy

${ }^{14}$ Department of Physics, Center for Cosmology and Astro-Particle Physics, The Ohio State University, Columbus, OH 43210, USA

${ }^{15}$ Istituto Nazionale di Fisica Nucleare, Sezione di Perugia, I-06123 Perugia, Italy

${ }^{16}$ Dipartimento di Fisica, Università degli Studi di Perugia, I-06123 Perugia, Italy

${ }^{17}$ Dipartimento di Fisica “M. Merlin” dell'Università e del Politecnico di Bari, I-70126 Bari, Italy

${ }^{18}$ Istituto Nazionale di Fisica Nucleare, Sezione di Bari, 70126 Bari, Italy

${ }^{19}$ Laboratoire Leprince-Ringuet, École polytechnique, CNRS/IN2P3, Palaiseau, France

${ }^{20}$ Department of Physics, University of Washington, Seattle, WA 98195-1560, USA

${ }^{21}$ Columbia Astrophysics Laboratory, Columbia University, New York, NY 10027, USA

${ }^{22}$ INAF-Istituto di Astrofisica Spaziale e Fisica Cosmica, I-20133 Milano, Italy ${ }^{23}$ NASA Goddard Space Flight Center, Greenbelt, MD 20771, USA

${ }^{24}$ Center for Research and Exploration in Space Science and Technology (CRESST), NASA Goddard Space Flight Center, Greenbelt, MD 20771, USA ${ }^{25}$ University of Maryland, Baltimore County, Baltimore, MD 21250, USA

${ }^{26}$ George Mason University, Fairfax, VA 22030, USA

${ }^{27}$ Laboratoire de Physique et Chemie de l'Environnement, LPCE UMR 6115 CNRS, F-45071 Orléans Cedex 02, and Station de radioastronomie de Nançay, Observatoire de Paris, CNRS/INSU, F-18330 Nançay, France

${ }^{28}$ Laboratoire de Physique Théorique et Astroparticules, Université Montpellier 2, CNRS/IN2P3, Montpellier, France

${ }^{29}$ Department of Physics and Astronomy, Sonoma State University, Rohnert Park, CA 94928-3609, USA

${ }^{30}$ Department of Physics, Stockholm University, AlbaNova, SE-106 91 Stockholm, Sweden

${ }^{31}$ Agenzia Spaziale Italiana (ASI) Science Data Center, I-00044 Frascati (Roma), Italy

${ }^{32}$ Dipartimento di Fisica, Università di Udine and Istituto Nazionale di Fisica Nucleare, Sezione di Trieste, Gruppo Collegato di Udine, I-33100 Udine, Italy ${ }^{33}$ Istituto Universitario di Studi Superiori (IUSS), I-27100 Pavia, Italy

${ }^{34}$ Santa Cruz Institute for Particle Physics, Department of Physics and Department of Astronomy and Astrophysics, University of California at Santa Cruz, Santa Cruz, CA 95064, USA 


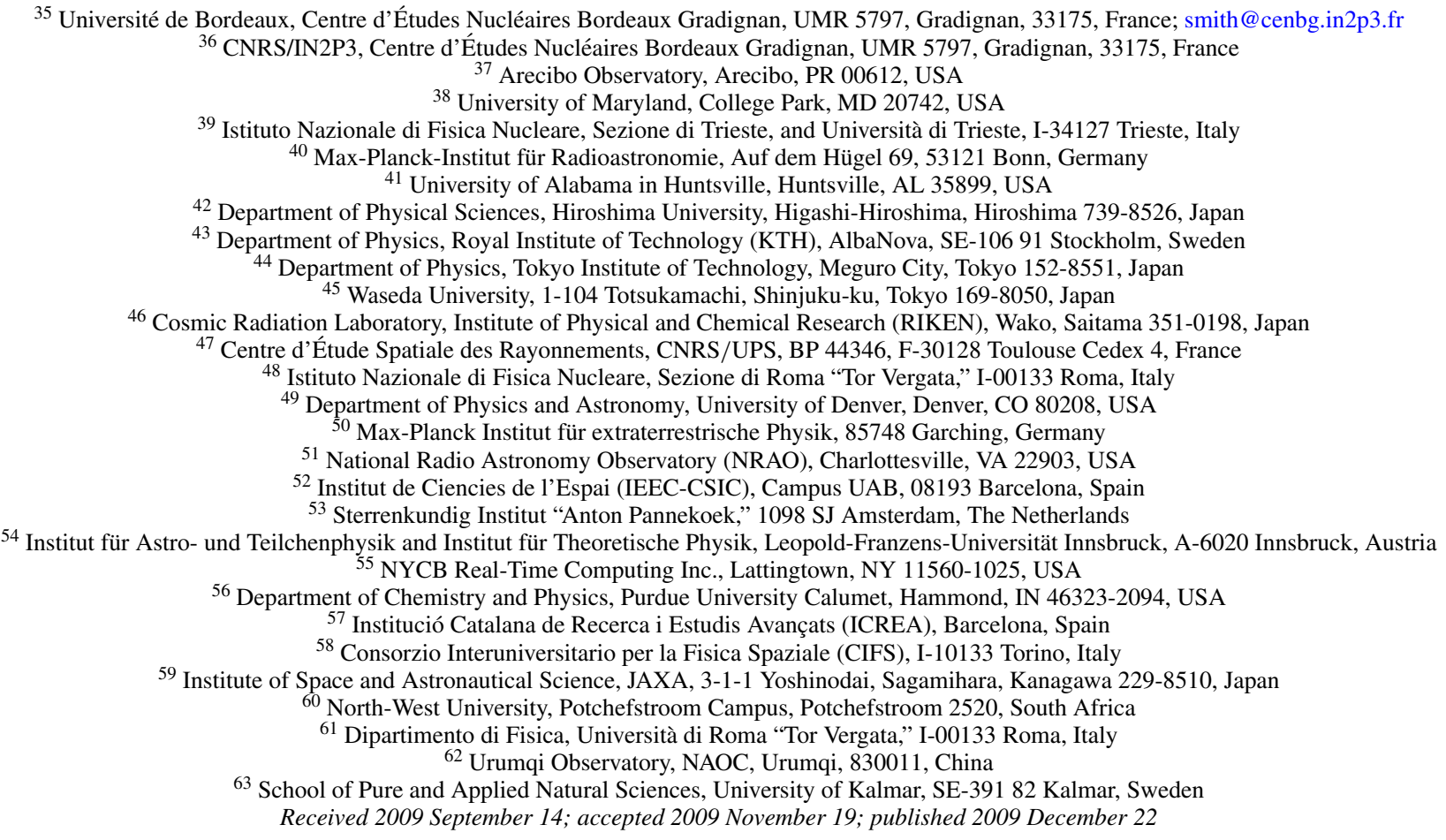

\section{ABSTRACT}

We report the detection of pulsed $\gamma$-rays for PSRs J0631+1036, J0659+1414, J0742-2822, J1420-6048, J15095850, and J1718-3825 using the Large Area Telescope on board the Fermi Gamma-ray Space Telescope (formerly known as GLAST). Although these six pulsars are diverse in terms of their spin parameters, they share an important feature: their $\gamma$-ray light curves are (at least given the current count statistics) single peaked. For two pulsars, there are hints for a double-peaked structure in the light curves. The shapes of the observed light curves of this group of pulsars are discussed in the light of models for which the emission originates from high up in the magnetosphere. The observed phases of the $\gamma$-ray light curves are, in general, consistent with those predicted by high-altitude models, although we speculate that the $\gamma$-ray emission of PSR J0659+1414, possibly featuring the softest spectrum of all Fermi pulsars coupled with a very low efficiency, arises from relatively low down in the magnetosphere. High-quality radio polarization data are available showing that all but one have a high degree of linear polarization. This allows us to place some constraints on the viewing geometry and aids the comparison of the $\gamma$-ray light curves with high-energy beam models.

Key words: pulsars: individual (PSRs J0631+1036, J0659+1414, J0742-2822, J1420-6048, J1509-5850, J1718-3825)

\section{INTRODUCTION}

The Fermi Gamma-ray Space Telescope (formerly known as GLAST) was successfully launched on 2008 June 11 . The study and discovery of $\gamma$-ray pulsars is one of the major goals of this mission. Studying pulsars at these high energies is important, because a large fraction of the total available spin-down energy loss rate $\left(\dot{E}=4 \pi^{2} I \dot{P} P^{-3}\right)$ is emitted in $\gamma$-rays. Here, $I$ is the moment of inertia of the star (generally taken to be $10^{45} \mathrm{~g} \mathrm{~cm}^{2}$ ), $P$ its spin period, and $\dot{P}$ its spin-down rate. By studying individual Fermi detections as well as the population of $\gamma$-ray pulsars as a whole, models for the high-energy emission can be constrained (e.g., Harding et al. 2007; Watters et al. 2009).

The models can be divided into three different families that place the emitting regions at different locations in the pulsar magnetosphere. In the so-called polar-cap model (e.g.,

\footnotetext{
${ }^{64}$ Royal Swedish Academy of Sciences Research Fellow, funded by a grant from the K. A. Wallenberg Foundation.
}

Daugherty \& Harding 1996), the $\gamma$-ray photons are produced close to the neutron star surface (within a few stellar radii) near the magnetic axis. At the other extreme are the outer-gap models (e.g., Morini 1983; Cheng et al. 1986; Romani \& Yadigaroglu 1995), which place the emitting region near the light cylinder. Finally, in slot-gap models (e.g., Muslimov \& Harding 2004), the particle acceleration occurs in a region bordering the last open field lines at a large range of emission altitudes. The two-pole caustic model (Dyks \& Rudak 2003) is a geometrical realization of the slot-gap model.

It has recently been demonstrated that $\gamma$-ray pulsars can be discovered via blind searches in the Fermi data (Abdo et al. 2009b). Nevertheless, the detection threshold for pulsed $\gamma$-rays is lower when accurate positions and spin frequencies (including their unpredictable timing irregularities) are already known. Therefore, a set of pulsars with $\dot{E}>10^{34} \mathrm{erg} \mathrm{s}^{-1}$ is being timed in the radio band, allowing Fermi to search for pulsations with the highest possible sensitivity (Smith et al. 2008). In addition, these radio observations allow us to determine the difference 
Table 1

Rotational and Derived Parameters for Six Pulsars

\begin{tabular}{ccccccc}
\hline \hline $\begin{array}{c}\text { PSR } \\
(\mathrm{J} 2000)\end{array}$ & $\begin{array}{c}\text { PSR } \\
(\mathrm{B} 1950)\end{array}$ & $\begin{array}{c}P \\
(\mathrm{~s})\end{array}$ & $\begin{array}{c}\dot{E} \\
\left(10^{35} \mathrm{erg} \mathrm{s}^{-1}\right)\end{array}$ & $\begin{array}{c}\tau_{\mathrm{c}}{ }^{\mathrm{a}} \\
\left(10^{3} \mathrm{yr}\right)\end{array}$ & $\begin{array}{c}B_{\mathrm{S}}{ }^{\mathrm{b}} \\
\left(10^{12} \mathrm{G}\right)\end{array}$ & $\begin{array}{c}B_{\mathrm{LC}}{ }^{\mathrm{c}} \\
\left(10^{3} \mathrm{G}\right)\end{array}$ \\
\hline $\mathrm{J} 0631+1036$ & & 0.288 & 1.73 & 43.6 & 5.55 & 2.18 \\
$\mathrm{~J} 0659+1414$ & $\mathrm{~B} 0656+14$ & 0.385 & 0.38 & 111 & 4.66 & 0.77 \\
$\mathrm{~J} 0742-2822$ & $\mathrm{~B} 0740-28$ & 0.167 & 1.43 & 157 & 1.69 & 3.43 \\
$\mathrm{~J} 1420-6048$ & & 0.068 & 104 & 13.0 & 2.41 & 71.3 \\
$\mathrm{~J} 1509-5850$ & & 0.089 & 5.15 & 154 & 9.14 & 12.2 \\
$\mathrm{~J} 1718-3825$ & & 0.075 & 12.5 & 89.5 & 1.01 & 22.6 \\
\hline
\end{tabular}

Notes.

a Spin-down age $\tau_{\mathrm{c}}=P /(2 \dot{P})$

b Magnetic field strength at the surface of the star in Gauss $B_{\mathrm{S}}=3.2 \times$ $10^{19}(P \dot{P})^{1 / 2}$.

c Magnetic field strength at the light cylinder in Gauss $B_{\mathrm{LC}}=3.0 \times$ $10^{8}\left(\dot{P} / P^{5}\right)^{1 / 2}$.

in arrival time of the $\gamma$-ray pulses with respect to the radio pulses, an important parameter to distinguish between different high-energy models.

In this paper, we report the detection of pulsed $\gamma$-rays for six pulsars that were found by folding the Fermi data on the ephemerides obtained from radio observations (e.g., Weltevrede et al. 2009). These Fermi detections will be included (although with more limited count statistics) in the Large Area Telescope (LAT) pulsar catalog paper (Abdo et al. 2009e). The six pulsars of this paper have moderate to large spin-down luminosities $\left(\dot{E}>10^{34.5} \mathrm{erg} \mathrm{s}^{-1}\right.$; see Table 1) and have (at least given the current count statistics) $\gamma$-ray light curves which are consistent with a single peak. Five of the six pulsars have a strong degree of linear polarization in the radio band, which can be used to constrain the emission geometry. Therefore the combination of radio and $\gamma$-ray data for these objects make them valuable for tests of the underlying beaming geometry.

The paper is organized such that we will start with describing the Fermi observations in Section 2. This is followed by a description of the methods used to constrain the emission geometry using radio data in Section 3. The results, including the $\gamma$-ray light curves and spectral parameters obtained with Fermi, are presented in Section 4 and discussed in Section 5. Finally, the results and conclusions are summarized in Section 6.

\section{OBSERVATIONS}

\subsection{Temporal Analysis}

The $\gamma$-ray data are collected by the LAT (Atwood et al. 2009), a pair-production telescope on board Fermi. With a large effective area ( $\sim 8000 \mathrm{~cm}^{2}$ above $1 \mathrm{GeV}$, on axis), a broad field of view ( $\sim 2.4$ steradian), and a high angular resolution, this telescope is far superior to the Energetic Gamma Ray Experiment Telescope (EGRET) on the Compton Gamma Ray Observatory (CGRO). The $\gamma$-ray events are time stamped using a GPS clock on board the satellite. These arrival times are transformed to a barycentric arrival time using the Fermi LAT Science Tools ${ }^{65}$ by taking into account the orbit of the satellite and the solar system ephemerides (Jet Propulsion Laboratory DE405; Standish 1998; Edwards et al. 2006). Tests have shown that the resulting precision is accurate to at least a few microseconds (Smith et al. 2008).

In this paper, we use photons which were collected between 2008 June 30 and 2009 May 22. Only so-called "diffuse" class

\footnotetext{
65 http://fermi.gsfc.nasa.gov/ssc/data/analysis/scitools/overview.html
}

Table 2

Parameters of the Analysis of the $\gamma$-ray Light Curves

\begin{tabular}{crrrr}
\hline \hline $\begin{array}{c}\text { PSR } \\
(\mathrm{J} 2000)\end{array}$ & $\begin{array}{c}\theta_{\max }{ }^{\mathrm{a}} \\
\left.{ }^{\circ}\right)\end{array}$ & \multicolumn{1}{c}{$H^{\mathrm{b}}$} & $\delta^{\mathrm{c}}$ & FWHM $^{\mathrm{d}}$ \\
\hline $\mathrm{J} 0631+1036$ & 1.0 & $9 \times 10^{-7}$ & $0.44 \pm 0.02 \pm 0.0002$ & 0.25 \\
$\mathrm{~J} 0659+1414$ & 1.0 & $<4 \times 10^{-8}$ & $0.21 \pm 0.01 \pm 0.002$ & 0.20 \\
$\mathrm{~J} 0742-2822$ & 1.0 & $9 \times 10^{-6}$ & $0.61 \pm 0.01 \pm 0.001$ & 0.10 \\
$\mathrm{~J} 1420-6048^{\mathrm{e}}$ & 0.5 & $<4 \times 10^{-8}$ & $0.36 \pm 0.02 \pm 0.01$ & 0.35 \\
$\mathrm{~J} 1509-5850^{\mathrm{e}}$ & 0.5 & $<4 \times 10^{-8}$ & $0.31 \pm 0.02 \pm 0.02$ & 0.40 \\
$\mathrm{~J} 1718-3825$ & 0.5 & $<4 \times 10^{-8}$ & $0.42 \pm 0.01 \pm 0.006$ & 0.20 \\
\hline
\end{tabular}

Notes.

${ }^{\text {a }}$ Maximum radius from the source to include $\gamma$-ray photons for the analysis.

${ }^{\mathrm{b}}$ Bin-independent $\mathrm{H}$-test probability that the pulsation would be caused by noise fluctuations.

${ }^{c}$ Peak position (phase lag) with respect to the peak of the radio profile (the uncertainties are the statistical and systematic errors, respectively).

${ }^{\mathrm{d}}$ Full width at half-maximum of the peak as a fraction of the pulse period.

e There is some evidence, although it is statistically not significant given the current count statistics, that the light curves of PSRs J1420-6048 and J1509-5850 consist of two overlapping components. If this interpretation is correct, then $\delta$ is 0.26 and 0.44 for the two peaks of PSR J1420-6048 and 0.18 and 0.39 for PSR J1509-5850.

Table 3

Radio Timing Parameters

\begin{tabular}{|c|c|c|c|}
\hline $\begin{array}{c}\text { PSR } \\
(\mathrm{J} 2000)\end{array}$ & $\mathrm{Obs}^{\mathrm{a}}$ & $\begin{array}{l}\mathrm{rms} \\
(\mu s)\end{array}$ & $\begin{array}{c}\mathrm{DM} \\
\left(\mathrm{cm}^{-3} \mathrm{pc}\right)\end{array}$ \\
\hline $\mathrm{J} 0631+1036$ & $\mathrm{~J} / \mathrm{N}$ & 51 & $125.36 \pm 0.01$ \\
\hline J0659+1414 & $\mathrm{P} / \mathrm{N}$ & 427 & $13.7 \pm 0.2$ \\
\hline J0742-2822 & $\mathrm{P} / \mathrm{J} / \mathrm{N}$ & 210 & $73.790 \pm 0.003$ \\
\hline $\mathrm{J} 1420-6048$ & $\mathrm{P}$ & 516 & $358.8 \pm 0.2$ \\
\hline J1509-5850 & $\mathrm{P}$ & 1068 & $140.6 \pm 0.8$ \\
\hline J1718-3825 & $\mathrm{P}$ & 434 & $247.88 \pm 0.09$ \\
\hline
\end{tabular}

Note. ${ }^{\text {a }}$ The radio observatories $(\mathrm{P}=$ Parkes, $\mathrm{J}=$ Jodrell Bank, and $\mathrm{N}=$ Nançay) involved in the radio timing.

events were used (Atwood et al. 2009), which are those with the highest probability to be caused by $\gamma$-rays from the source. All $\gamma$-rays events with a reconstructed zenith angle larger than $105^{\circ}$ were ignored to avoid the intense $\gamma$-ray background caused by cosmic-ray interactions in the Earth's atmosphere.

To produce the $\gamma$-ray light curves, we used photons within an energy-dependent radius $\theta \leqslant 0.8 \times E_{\mathrm{GeV}}^{-0.75}$ degrees of the pulsar position, requiring a radius of at least 0.35 , but not larger than the $\theta_{\max }$ of 0.5 or 1.0 as shown in Table 2. See the LAT pulsar catalog paper (Abdo et al. 2009e) for a detailed discussion of the selection criteria. This selection maximizes the signal-tonoise ratio $(\mathrm{S} / \mathrm{N})$ over the broad energy range covered by the LAT. In all cases, the background is estimated from off-pulse bins from a $1^{\circ}-2^{\circ}$ ring around the pulsar using the same energydependent cut.

The pulsars discussed in this paper are regularly observed in the radio band near $1.4 \mathrm{GHz}$ by the Fermi Timing Consortium (see Table 3). The observatories involved are the Parkes 64-m radio telescope in Australia, the Lovell 76-m telescope at the Jodrell Bank observatory near Manchester in England, and the 94-m (equivalent) Nançay radio telescope near Orleans, France. The timing program at Parkes is described in Weltevrede et al. (2009) and typically involves monthly observations. Nançay (Cognard et al. 2009) and Jodrell Bank (Hobbs et al. 2004) observations are made, on average, every 5-9 days. 
The times of arrival (TOAs) for PSRs J1420-6048, J15095850 , and J1718-3825 were obtained solely from Parkes data. For the other pulsars, the TOAs obtained from the different telescopes were combined before making an ephemeris for the spin behavior of the neutron star. The TOAs were compared with an initial timing solution using TEMPO2 (Hobbs et al. 2006) producing timing residuals which then were fit for the spinfrequency and its time derivative, as well as for instrumental offsets between data from different observatories. Most of the pulsars showed strong additional deviations in their spin behavior, known as timing noise (e.g., Hobbs et al. 2004). This timing noise is modeled by either adding higher order spinfrequency derivatives or by using the fitwaves algorithm within TEMPO2.

The resulting timing model allows an accurate assignment of a rotational phase to the $\gamma$-ray photons (using TEMPO2), thereby constructing the light curves. The timing parameters used in this work will be made available on the servers of the Fermi Science Support Center. ${ }^{66}$

An important parameter necessary to align the radio profile with the $\gamma$-ray light curve is the dispersion measure (DM), which quantifies the frequency-dependent delay of the radio emission caused by the interstellar medium. The DM of PSR J0631+1036 was measured by comparing the TOAs at two widely separated frequencies using Jodrell Bank data $(0.6 \mathrm{GHz}$ and $1.4 \mathrm{GHz})$ after the templates used were carefully aligned so that the components at the two frequencies coincide in pulse phase. The DM of the other five pulsars were obtained by measuring the delay across the $256 \mathrm{MHz}$ band using Parkes data (see Weltevrede et al. 2009 and Table 3). The systematic error in the alignment of the radio and the $\gamma$-ray light curves is the combination of the DM uncertainty and the rms scatter of the radio timing residuals.

The shapes of the light curves (consisting of all photons above $100 \mathrm{MeV}$ ) were fitted using Gaussian functions resulting in peak positions and the error bars on the light curves were taken to be the square root of the number of photons in each phase bin. The results of the fitting of the light curves together with the full width at half-maximum (FWHM) estimated by eye are summarized in Table 2. This table includes the bin-independent H-test probability (de Jager et al. 1989), which measures the probability that the observed $\gamma$-ray light curves are caused by noise fluctuations.

\subsection{Spectral Analysis}

The spectral analysis uses the same first six months of Fermi data as the LAT pulsar catalog (Abdo et al. 2009e). The LAT "gtlike" science tool ${ }^{67}$ performs a maximum likelihood analysis (Mattox et al. 1996) to fit phase-averaged spectra for the six pulsars. Instrument Response Functions (IRFs) allow proper treatment of the direction and energy of each event. We used "Pass 6 v3," a post-launch IRF update that addresses inefficiencies correlated with the trigger rate ${ }^{67}$ Angular resolution is poor at low energies: at $100 \mathrm{MeV}$ and normal detector incidence, $68 \%$ of the photons from a point source have reconstructed directions within $\sim 5^{\circ}$ of the true direction, decreasing to $\sim 0.2$ at $10 \mathrm{GeV}$. Therefore, the likelihood analysis must model not just the pulsar under study, but all neighboring $\gamma$-ray sources as well. We applied the analysis used for the three-month Fermi Bright Source List (BSL; Abdo et al. 2009d) with an updated model for the Galactic diffuse emission to this six-month data set. Then, as

\footnotetext{
66 http://fermi.gsfc.nasa.gov/ssc/data/access/lat/ephems/

$67 \mathrm{http}$ ://fermi.gsfc.nasa.gov/ssc/data/analysis/documentation/
}

Table 4

The Parameters of the Spectral Analysis of the $\gamma$-ray Data ${ }^{a}$

\begin{tabular}{ccccc}
\hline \hline $\begin{array}{c}\text { PSR } \\
(\mathrm{J} 2000)\end{array}$ & $\begin{array}{c}\text { Energy Flux }(E>100 \mathrm{MeV}) \\
\left(10^{-11} \mathrm{erg} \mathrm{cm}^{-2} \mathrm{~s}^{-1}\right)\end{array}$ & $\Gamma$ & $\begin{array}{c}E_{\text {cutoff }} \\
(\mathrm{GeV})\end{array}$ & $\sigma_{\text {cutoff }}$ \\
\hline $\mathrm{J} 0631+1036$ & $3.04 \pm 0.51$ & $1.38 \pm 0.35$ & $3.6 \pm 1.8$ & 3.2 \\
$\mathrm{~J} 0659+1414$ & $3.17 \pm 0.31$ & $2.37 \pm 0.42$ & $0.7 \pm 0.5$ & 2.6 \\
$\mathrm{~J} 0742-2822$ & $1.83 \pm 0.36$ & $1.76 \pm 0.40$ & $2.0 \pm 1.4$ & 2.0 \\
$\mathrm{~J} 1420-6048$ & $15.9 \pm 2.8$ & $1.73 \pm 0.20$ & $2.7 \pm 1.0$ & 4.6 \\
$\mathrm{~J} 1509-5850$ & $9.7 \pm 1.0$ & $1.36 \pm 0.23$ & $3.5 \pm 1.1$ & 5.1 \\
$\mathrm{~J} 1718-3825$ & $6.8 \pm 1.7$ & $1.26 \pm 0.62$ & $1.3 \pm 0.6$ & 4.4 \\
\hline
\end{tabular}

Note. ${ }^{\text {a }}$ The spectral analysis is based on the first six months of Fermi data as presented in the LAT pulsar catalog paper (Abdo et al. 2009e).

for the BSL, we extract events in a circle of radius $10^{\circ}$ around each pulsar. The likelihood model includes all sources up to $17^{\circ}$ from each pulsar, with spectral parameters fixed to the values obtained from the BSL analysis for those more than $3^{\circ}$ away. Spectral parameters for the pulsar, as well as for sources within $3^{\circ}$, are left free in the fit. Galactic diffuse emission was modeled using a GALPROP (Strong et al. 2004) calculation designated $54 \_77$ Xvarh7S, very similar to that available from the Fermi Science Support Center. ${ }^{67}$

Bright $\gamma$-ray pulsars like Vela (Abdo et al. 2009c) or the Crab (Abdo et al. 2009a) are observed to have spectra which are well described by exponentially cutoff power-law models of the form

$$
\frac{\mathrm{d} N}{\mathrm{~d} E}=K E_{\mathrm{GeV}}^{-\Gamma} \exp \left(-\frac{E}{E_{\text {cutoff }}}\right),
$$

in which the three parameters are the normalization $K$ of the differential flux (in units of $\mathrm{ph} \mathrm{cm}^{-2} \mathrm{~s}^{-1} \mathrm{MeV}^{-1}$ ), the spectral index, $\Gamma$, and the cutoff energy, $E_{\text {cutoff }}$. The energy at which the differential flux is defined is arbitrary. We choose $1 \mathrm{GeV}$ because it is, for most pulsars, close to the energy at which the relative uncertainty on the differential flux is minimal. The spectra were fitted separately using a power law and a power law plus exponential cutoff. The difference $\Delta$ of the $\log$ (likelihood) for the two fits determines the significance $\sigma_{\text {cutoff }}$ for the existence of an energy cutoff, which is defined to be the test statistic difference $\sqrt{2 \Delta}$. For pulsars with a $\sigma_{\text {cutoff }}<3$, the power law with a cutoff did not result in a significantly better fit compared to a simple power law, hence the cutoff energy is unconstrained.

The observed $\gamma$-ray energy flux $F_{\text {obs }}$ is the integral above $100 \mathrm{MeV}$ of the fitted spectral shape times the energy. The luminosity is then

$$
L_{\gamma}=4 \pi f_{\Omega} F_{\mathrm{obs}} D^{2},
$$

where $D$ is the distance and $f_{\Omega}$ is the flux correction factor which depends on the beaming fraction (e.g., Watters et al. 2009). For outer magnetospheric models, $f_{\Omega}$ is thought to be $\sim 1$, which is the value we assume throughout this paper except in Table 5. This luminosity can then be compared to the spin-down energy loss rate of the pulsar to obtain the $\gamma$-ray efficiency

$$
\eta=L_{\gamma} / \dot{E} .
$$

The results of the spectral analysis are summarized in Table 4 and the derived luminosities and efficiencies can be found in Table 5. Note that the distances to all the pulsars except PSR J0659+1414 are highly uncertain, as described in more detail below.

Uncertainties on the effective area $(\leqslant 5 \%$ near $1 \mathrm{GeV}, 10 \%$ below $0.1 \mathrm{GeV}$, and $20 \%$ over $10 \mathrm{GeV}$ ) and uncertainties in the 
Table 5

The $\gamma$-ray Luminosity and the Efficiency, which are Functions of the Flux Correction Factor, $f_{\Omega}$, and the Pulsar Distance, $D$

\begin{tabular}{ccc}
\hline \hline $\begin{array}{c}\text { PSR } \\
(\mathrm{J} 2000)\end{array}$ & $\begin{array}{c}L_{\gamma} \\
\left(10^{35} \mathrm{erg} \mathrm{s}^{-1}\right)\end{array}$ & $\eta$ \\
\hline $\mathrm{J} 0631+1036$ & $(0.036 \pm 0.006) f_{\Omega}(D / 1 \mathrm{kpc})^{2}$ & $(0.021 \pm 0.004) f_{\Omega}(D / 1 \mathrm{kpc})^{2}$ \\
$\mathrm{~J} 0659+1414$ & $(0.0032 \pm 0.0003) f_{\Omega}(D / 0.288 \mathrm{kpc})^{2}$ & $(0.0084 \pm 0.0008) f_{\Omega}(D / 0.288 \mathrm{kpc})^{2}$ \\
$\mathrm{~J} 0742-2822$ & $(0.09 \pm 0.02) f_{\Omega}(D / 2 \mathrm{kpc})^{2}$ & $(0.06 \pm 0.01) f_{\Omega}(D / 2 \mathrm{kpc})^{2}$ \\
$\mathrm{~J} 1420-6048$ & $(6 \pm 1) f_{\Omega}(D / 5.6 \mathrm{kpc})^{2}$ & $(0.06 \pm 0.01) f_{\Omega}(D / 5.6 \mathrm{kpc})^{2}$ \\
$\mathrm{~J} 1509-5850$ & $(0.75 \pm 0.08) f_{\Omega}(D / 2.5 \mathrm{kpc})^{2}$ & $(0.15 \pm 0.02) f_{\Omega}(D / 2.5 \mathrm{kpc})^{2}$ \\
$\mathrm{~J} 1718-3825$ & $(1.1 \pm 0.3) f_{\Omega}(D / 3.6 \mathrm{kpc})^{2}$ & $(0.09 \pm 0.03) f_{\Omega}(D / 3.6 \mathrm{kpc})^{2}$ \\
\hline
\end{tabular}

Galactic diffuse emission model dominate the systematic uncertainties on the spectral results as described in the LAT pulsar catalog (Abdo et al. 2009e). The spectral parameter uncertainties are $\delta \Gamma=(+0.3,-0.1), \delta E_{\text {cutoff }}=(+20 \%,-10 \%)$, and $\delta F_{\text {obs }}=(+20 \%,-10 \%)$. The bias on the integral energy flux is somewhat less than that of the integral photon flux, due to the weighting by photons in the energy range where the effective area uncertainties are smallest. We do not sum these uncertainties in quadrature with the others, since a change in instrument response will tend to shift all spectral parameters similarly.

\section{DERIVING EMISSION GEOMETRIES FROM RADIO DATA}

Two important angles used to describe the emission geometry of pulsars are the angle $\alpha$ between the magnetic axis and the rotation axis and the angle $\zeta$ between the line of sight and the rotation axis. A related angle is the impact parameter $\beta=\zeta-\alpha$, which is the angle between the line of sight and the magnetic axis at its closest approach. These angles can be inferred by applying the rotating vector model (RVM; Radhakrishnan \& Cooke 1969) to the position angle (P.A.) of the linear polarization observed in the radio band. This model predicts the P.A. of the linear polarization $\psi$ to depend on the pulse phase $\phi$ as

$\tan \left(\psi-\psi_{0}\right)=\frac{\sin \alpha \sin \left(\phi-\phi_{0}\right)}{\sin \zeta \cos \alpha-\cos \zeta \sin \alpha \cos \left(\phi-\phi_{0}\right)}$,

where $\psi_{0}$ and $\phi_{0}$ are the P.A. and pulse phase corresponding to the intersection of the line of sight with the fiducial plane (the plane containing the rotation and magnetic axis) if the emission height $h_{\mathrm{em}}$ is small compared to the light cylinder distance. In this model, the P.A.-swing is an S-shaped curve and its inflection point occurs at $\phi_{0}$.

It is found that the degree of linear polarization is correlated with $\dot{E}$ such that virtually all pulsars with $\dot{E}>2 \times 10^{35} \mathrm{erg} \mathrm{s}^{-1}$ have a linear polarization fraction over $50 \%$ (Weltevrede \& Johnston 2008; see also Qiao et al. 1995; von Hoensbroech et al. 1998; Crawford et al. 2001; Johnston \& Weisberg 2006). Moreover, most of these pulsars have smooth P.A.-swings, making it relatively easy to apply the RVM model to the pulsars presented in this paper.

If the emission profile is symmetric around the magnetic axis, then the inflection point coincides with the middle of the pulse profile. However, co-rotation of the emitting region causes the inflection point to be delayed with respect to the pulse profile. This pulse phase difference $\Delta \phi$ between the middle of the profile and the inflection point of the P.A.-swing can be used to estimate the emission height (Blaskiewicz et al. 1991)

$$
h_{\mathrm{P} . \mathrm{A} .}=\frac{P c}{8 \pi} \Delta \phi=\frac{1}{4} R_{\mathrm{LC}} \Delta \phi,
$$

where $P$ is the spin period of the pulsar, $c$ is the speed of light, and $R_{\mathrm{LC}}$ is the light cylinder radius. Because the relative shift of the P.A.-swing with respect to the profile is independent of $\alpha$ and $\zeta$ (Dyks et al. 2004), Equation (4) can be used to fit the P.A.-swing even for moderate emission heights (Dyks 2008). Use of these equations ignores effects of rotational sweepback of the magnetic field lines (Dyks \& Harding 2004), propagation effects in the pulsar magnetosphere (e.g., Petrova 2006), currentinduced distortions of the magnetic field and the effects of a finite emission height spread and emission height differences (Dyks 2008).

Both the P.A.-swing and the observed pulse width contain information about the geometry of the pulsar. Under the assumption that the radio beam is symmetric about the magnetic axis, the pulse width $W$ (which we take to be the full phase range over which we see emission) is related to the half opening angle $\rho$ of the beam via

$$
\cos \rho=\cos \alpha \cos \zeta+\sin \alpha \sin \zeta \cos (W / 2),
$$

(Gil et al. 1984; Lorimer \& Kramer 2005). Therefore, if we know the value of $\rho$ for a given pulsar, Equation (6) can be used as an additional constraint on Equation (4) to narrow down the allowed region in $\alpha-\beta$ space. The value of $\rho$ can be estimated from the emission height via

$$
\rho=\sqrt{\frac{9 \pi h_{\mathrm{em}}}{2 P c}},
$$

assuming a radio beam centred about the magnetic axis which is enclosed by the last open dipole field lines (e.g., Lorimer \& Kramer 2005). In general, although there is some evidence for conal rings centered on the magnetic axis (Rankin 1983), many pulsars are patchy (e.g., Lyne \& Manchester 1988; Han \& Manchester 2001; Keith et al. 2009; Weltevrede \& Wright 2009), making both $\rho$ and $h_{\text {P.A. }}$ uncertain. This uncertainty is likely to dominate the total uncertainty of the radio analysis, and its impact is discussed for the individual pulsars in the next section. Because of poorly understood systematics, it is impossible to come up with sensible error bars on the derived values describing the geometry.

In summary, first we can use the RVM to fit the P.A.-swing (Equation (4)), resulting in contours defining the allowed $\alpha-\beta$ parameter space. Secondly, from the offset of the P.A.-swing with respect to the total intensity profile, we can estimate an emission height (Equation (5)), which can be translated to an opening angle of the radio beam (Equation (7), assuming $h_{\mathrm{em}}=h_{\text {P.A. }}$ ). This opening angle corresponds to another contour in $\alpha-\beta$ parameter space (Equation (6)), which in the ideal case would match with the RVM contours. If not, then at least one of the assumptions must be incorrect, most likely indicating that the radio beam is asymmetric with respect to the magnetic axis. 
Additional constraints on the emission geometry can be obtained from measurements of the termination shock of the surrounding pulsar wind nebula (PWN), which can provide a relatively model-independent estimate of the viewing angle $\zeta(\mathrm{Ng}$ \& Romani 2008); unfortunately, such measurements are not yet available for the pulsars discussed here.

\section{RESULTS ON THE INDIVIDUAL PULSARS}

\subsection{PSR J0631+1036}

\subsubsection{The Pulsar and its Surroundings}

PSR J0631+1036 was discovered as a young pulsar by Zepka et al. (1996) in a radio search targeting Einstein IPC X-ray sources. Its DM is very high for a pulsar in the Galactic anticenter and this is argued to be caused by the foreground star-forming region 3 Mon (Zepka et al. 1996). In addition, this pulsar could be interacting with (or be embedded in) dark cloud LDN 1605 and therefore the distance derived from the DM ( $3.6 \pm 1.3 \mathrm{kpc}$, according to the Cordes \& Lazio 2002 model) is possibly overestimated. Following Zepka et al. (1996), we adopt a distance of $1 \mathrm{kpc}$ consistent with the observed X-ray absorption. No PWN has been found for this pulsar at radio wavelengths (Gaensler et al. 2000).

$$
\text { 4.1.2. } \gamma \text {-rays }
$$

PSR J0631+1036 clearly shows $\gamma$-ray pulsations (see Figure 1) and the light curve features a single broad peak (FWHM 0.25 in rotational phase), which lags the radio profile by 0.44 (see Table 2). A power law in combination with an exponential cutoff fits the $\gamma$-ray spectrum significantly better than a single power law, although the cutoff energy cannot be accurately determined.

Zepka et al. (1996) claimed a $\gamma$-ray detection by EGRET of PSR J0631+1036. The detection was very marginal and their light curve does not really resemble that seen by Fermi. Their estimated $\gamma$-ray flux is an order of magnitude larger than that obtained from the Fermi data (see Table 4).

\subsubsection{X-rays}

At the pulsar position a faint ROSAT PSPC X-ray source has been found, which was too weak to search for pulsations (Zepka et al. 1996). Sinusoidal X-ray pulsations were claimed by Torii et al. (2001) using ASCA data. However, an XMMNewton observation appears to show that the $\mathrm{X}$-ray point source is not associated with the pulsar and the pulsations were not confirmed (Kennea et al. 2002). The derived upper limit for a $\mathrm{X}$-ray point source is $1.1 \times 10^{30} \mathrm{erg} \mathrm{s}^{-1}(0.5-2.0 \mathrm{keV})$ for the assumed distance of $1 \mathrm{kpc}$. This luminosity is low compared to the Becker \& Trümper (1997) relationship between the X-ray luminosity and $\dot{E}$, which can be seen as evidence for a larger distance to the pulsar.

\subsubsection{Radio}

The pulsar's radio spectrum is relatively flat from 1.4 up to at least $6.2 \mathrm{GHz}$ (unpublished Parkes data ${ }^{68}$ ) and its radio profile is highly polarized (Zepka et al. 1996; Weltevrede \& Johnston 2008). The radio profile consists of four components (see Figure 2) and the outer components are strongest at low frequencies. The radio profile shows a remarkable deep minimum at the pulse phase of the symmetry point. Despite the

\footnotetext{
68 www.atnf.csiro.au/people/joh414/ppdata
}
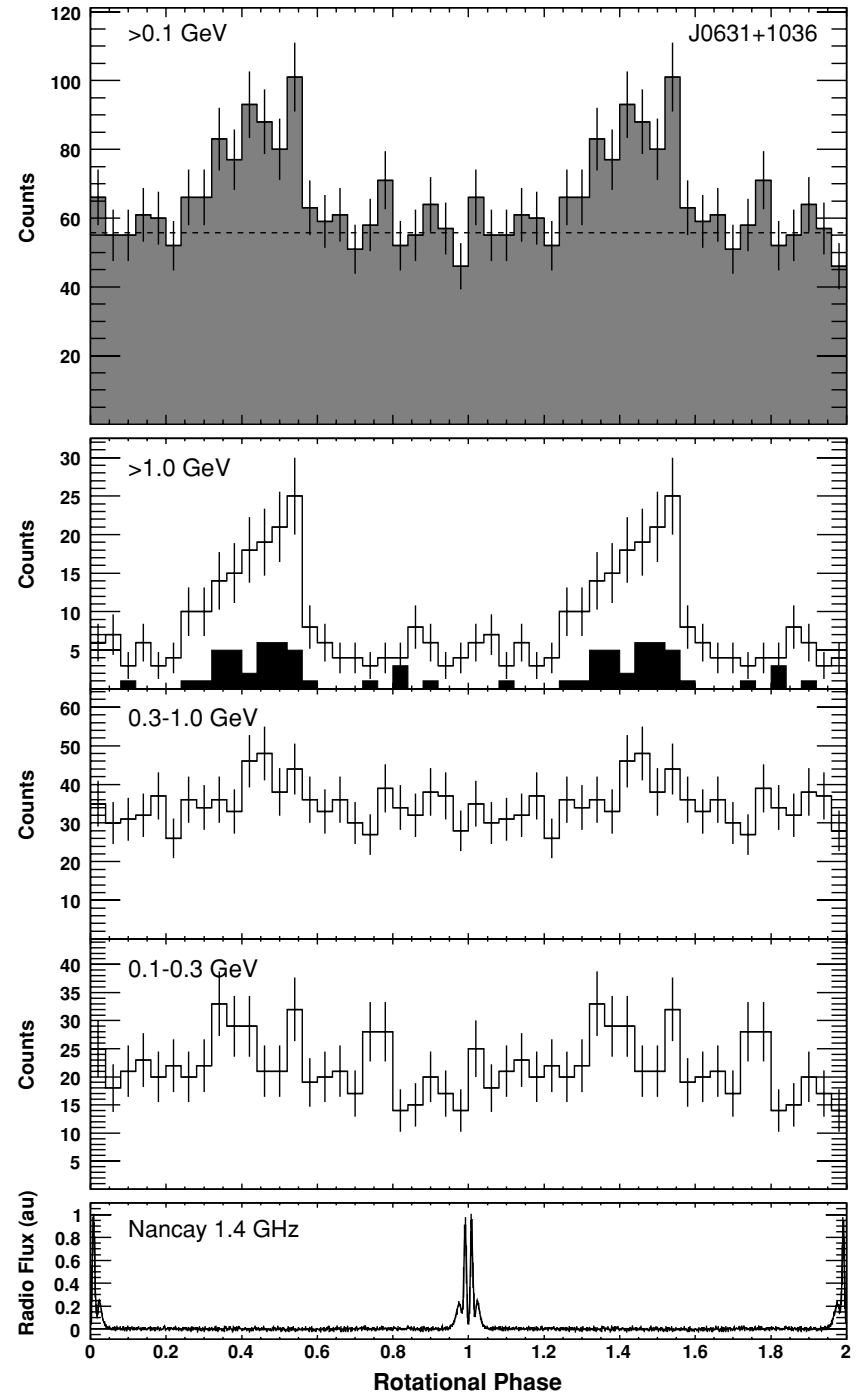

Figure 1. Fermi $\gamma$-ray light curves of PSR J0631+1036 in different energy bands showing two full rotational periods with 25 bins per period. The photons above $3 \mathrm{GeV}$ are shown in black in the second panel from the top. The background estimate is shown as a dashed line in the top panel. The bottom panel shows the phase-aligned radio profile.

complex structure of the profile, it is highly mirror symmetric, not only in its shape, but also in the spectral indices of the different components.

We fitted the RVM model (Equation (4)) to the radio polarization data. The resulting $\chi^{2}$ map is shown in gray scale and in the black contours in Figure 3. One can see a "banana-shaped" region in $\alpha-\beta$ space, which contains valid solutions for the P.A.-swing, showing that $\alpha$ is not constrained.

The remarkable degree of mirror symmetry of the radio profile can be seen as evidence that the radio beam itself has a high degree of symmetry and is centered at the magnetic axis. Under this assumption, the radio emission height follows from the offset of the steepest gradient of the P.A. swing and the mirror point of the profile (Equation (5)) and is found to be $h_{\text {P.A. }}=600 \mathrm{~km}$, which is slightly lower than found by Weltevrede \& Johnston (2008) using data with a lower S/N. Using this emission height in Equation (7), we expect a half opening angle of the beam $\rho$ of $18^{\circ}$ if the emission comes from the last open field line. In Figure 3, the contours of $\rho$ derived from the observed pulse width are overplotted (dotted). One can 


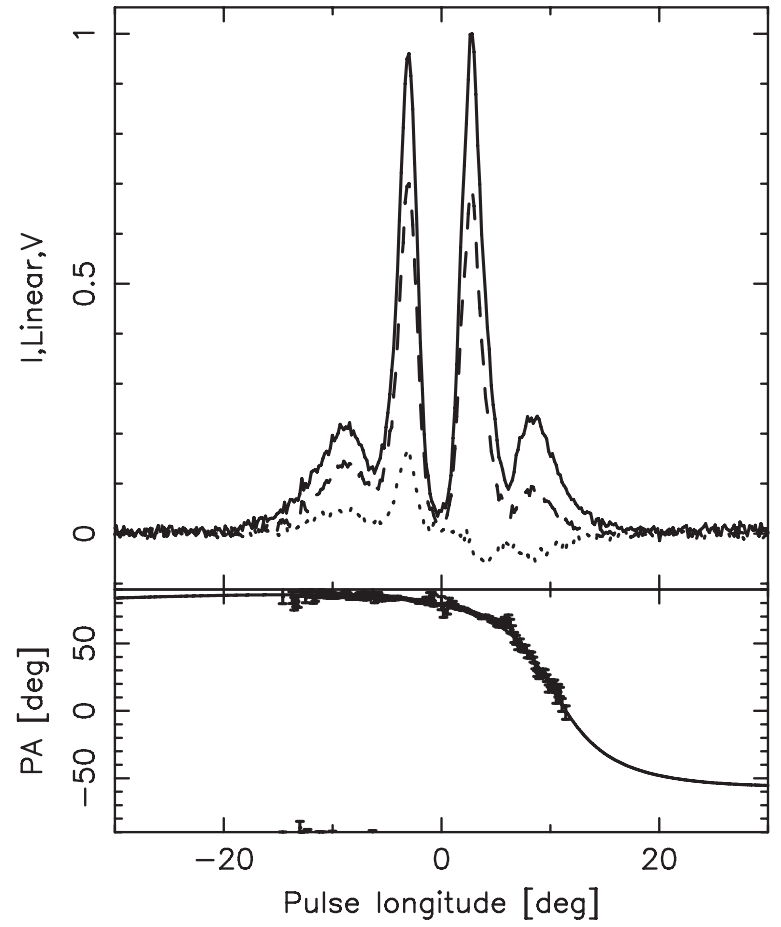

Figure 2. Pulse profile at $1398 \mathrm{MHz}$ observed at Nançay (black) of PSR J0631+1036, as well as the degree of linear polarization (dashed) and circular polarization (dotted). The bottom panel shows the P.A. of the linear polarization (if detected above $3 \sigma$ ) and an RVM fit.

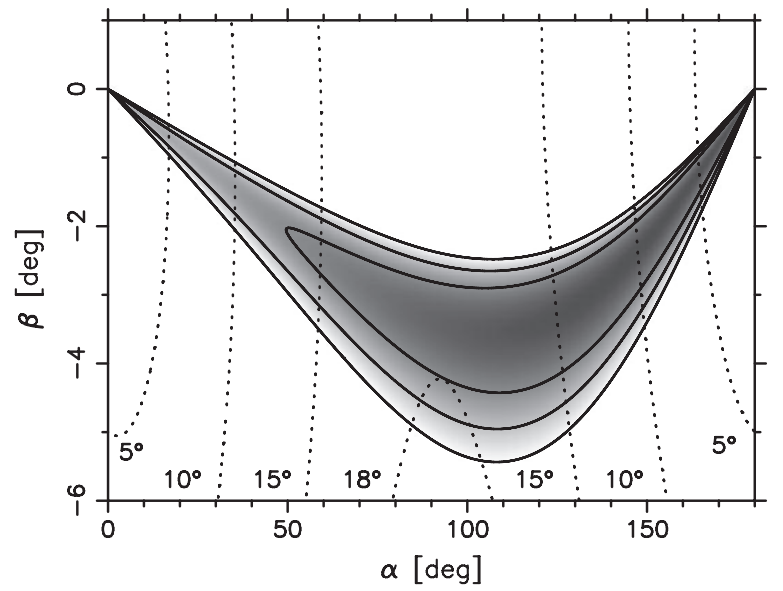

Figure 3. $\chi^{2}$ map of the RVM fit of J0631+1036 (in gray scale, a darker color indicates a lower $\chi^{2}$ ). The lowest reduced $\chi^{2}$ is 1.45 and the solid contours correspond to reduced $\chi^{2}$ values that are two, three, and four times larger. The half opening angles $\rho$ of the radio beam derived from the observed pulse width are overplotted (dotted contours).

see that such a large value of $\rho$ suggests that $\alpha$ is close to $90^{\circ}$ and $\beta \sim-4^{\circ}$. If only a fraction of the open field lines produces radio emission (i.e., if the beam is patchy), then $\alpha$ could be smaller.

\subsection{PSR J0659+1414 (B0656+14)}

\subsubsection{The Pulsar and its Surroundings}

PSR J0659+1414 was discovered in the second Molonglo pulsar survey as a radio pulsar (Manchester et al. 1978) and this pulsar is the slowest rotating and has the lowest $\dot{E}$ of the pulsars discussed in this paper. The distance of the pulsar is well known via parallax measurements using very long
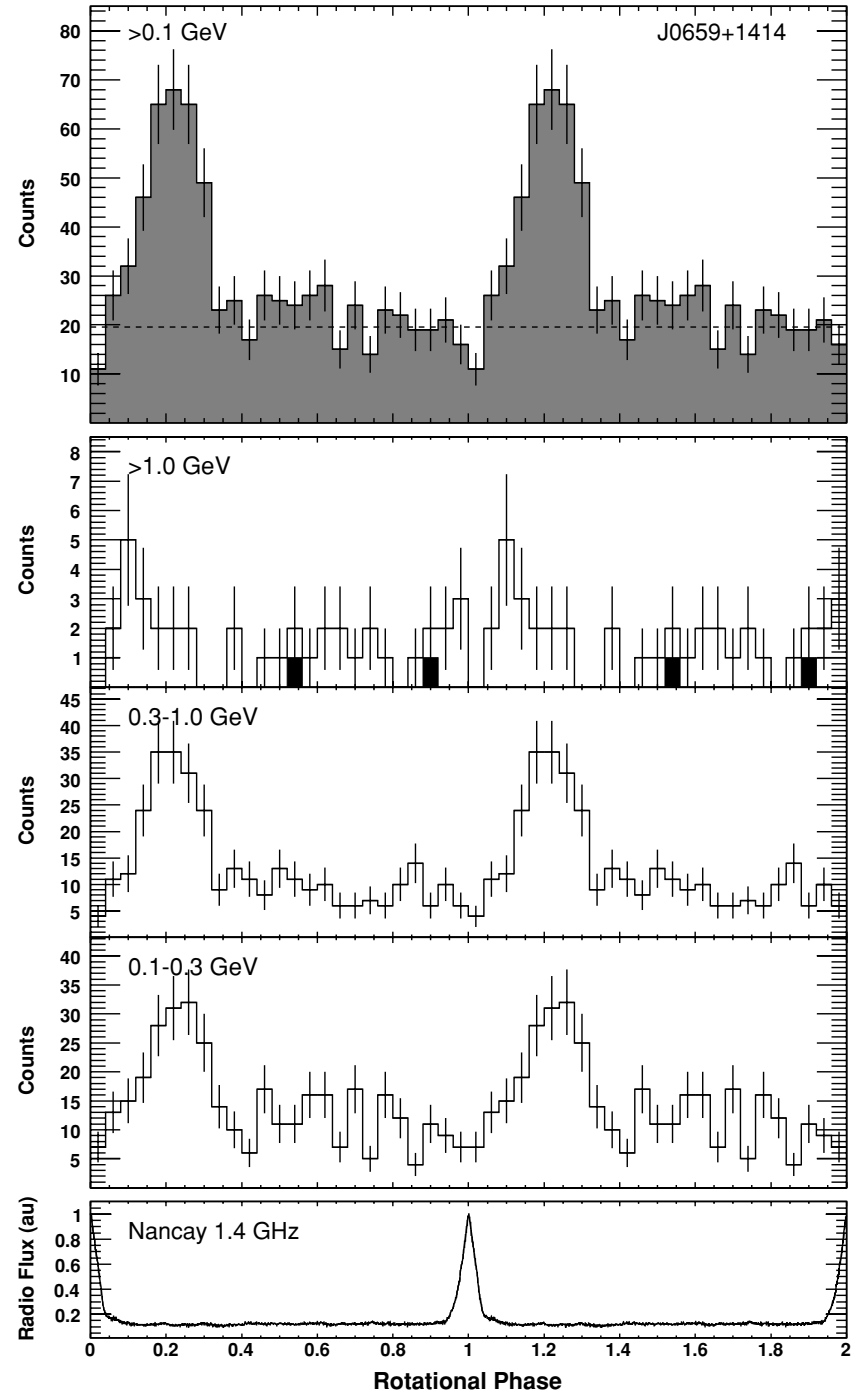

Figure 4. As in Figure 1, but for PSR J0659+1414.

baseline interferometry (288 $8_{-27}^{+33}$ pc; Brisken et al. 2003). PSR J0659+1414 is associated with the Monogem ring (Thorsett et al. 2003), a bright diffuse $25^{\circ}$ diameter supernova remnant easily visible in soft X-ray images of the sky. It has a possible PWN in optical (Shibanov et al. 2006) and X-rays (Marshall \& Schulz 2002).

\subsection{2. $\gamma$-rays}

There was a marginal detection of pulsed $\gamma$-rays from this pulsar by EGRET (Ramanamurthy et al. 1996). The light curve observed by Fermi (see Figure 4) is similar to that seen by EGRET, but the $\gamma$-ray background is much lower in the Fermi data because of its superior angular resolution. The light curve is single peaked (FWHM 0.20 in rotational phase) and lags the radio peak by 0.21 in phase.

The pulsar is very weak above $1 \mathrm{GeV}$, showing that its spectrum is extremely soft. Indeed this pulsar appears to have the steepest spectrum and the lowest cutoff energy of the pulsars in this paper (see Table 4). Although the cutoff appears to be within the energy range of the LAT detector, a power law plus exponential cutoff does not describe the data better than a single power law. The $\gamma$-ray efficiency of PSR J0659+1414 is very low (see Table 5). Because the distance to the pulsar 


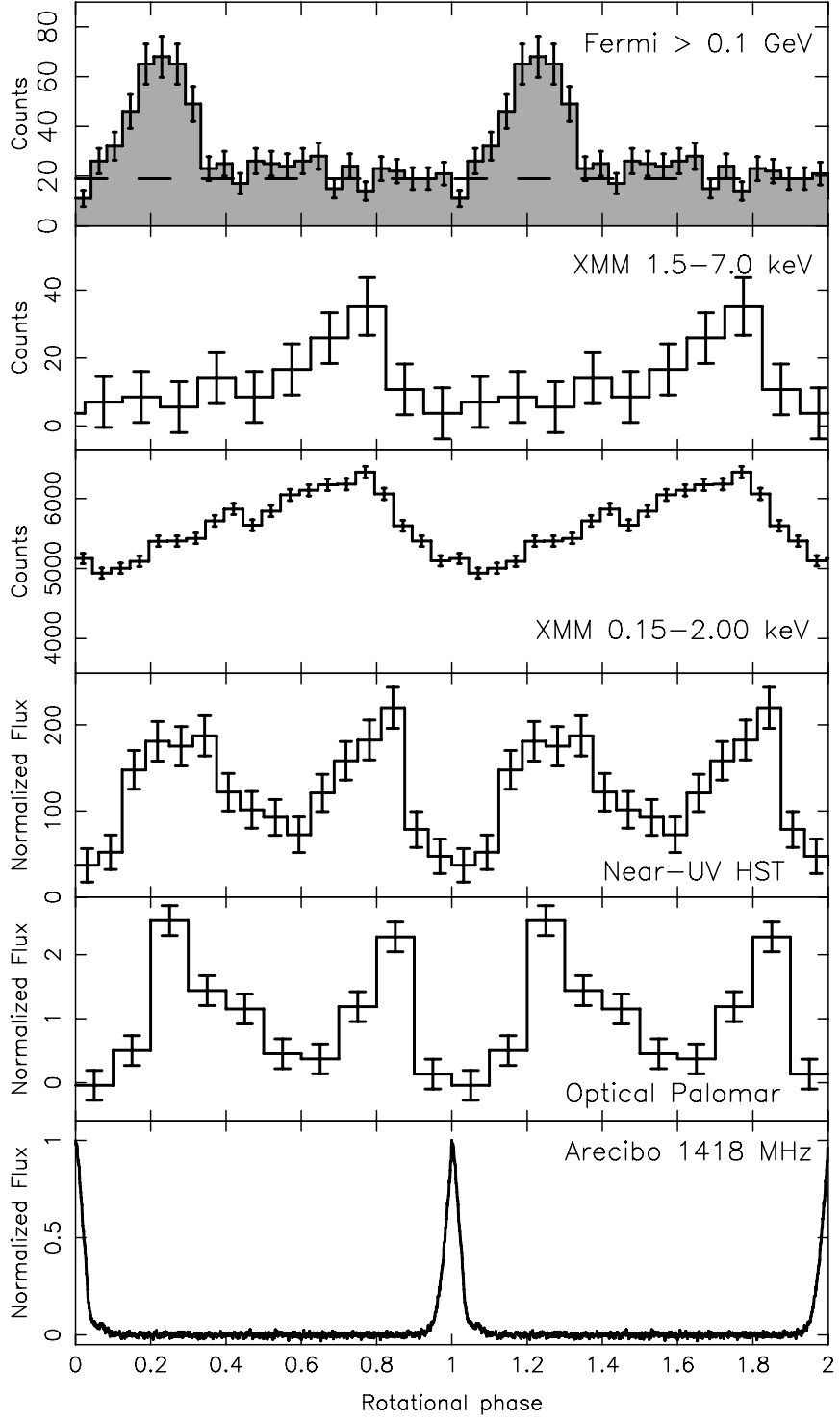

Figure 5. Phase-aligned light curves of PSR J0659+1414 at multiple wavelengths. The Fermi light curve (with its background level indicated by the dashed line) is compared with the XMM-Newton observations (De Luca et al. 2005), shown in two energy ranges encompassing thermal (0.15-2 keV) and non-thermal (1.5-7 keV) components (note that for the lower panel the vertical axis does not start at zero, indicating a large unpulsed fraction of the emission). The near-UV light curve is from Shibanov et al. (2005), who aligned the light curve by making use of the similarity with the optical light curve. The background-subtracted optical light curve is from Kern et al. (2003), and the radio profile is from Everett \& Weisberg (2001).

is well determined, the low efficiency cannot be caused by an incorrectly estimated luminosity.

\subsubsection{X-rays}

PSR J0659+1414 is one of the brightest isolated neutron stars in the X-ray sky (Córdova et al. 1989), and it is one of the "Three Musketeers" (the others being Geminga and PSR B1055-52; Becker \& Trümper 1997). The X-ray emission is a combination of thermal (blackbody) and non-thermal (power law) emission (e.g., De Luca et al. 2005) and is consistent with a cooling middle-aged neutron star (e.g., Becker \& Trümper 1997). At soft X-rays, the pulse fraction is low and the pulsations are sinusoidal (see Figure 5), typical for thermal emission from the surface of a neutron star with a non-uniform temper-

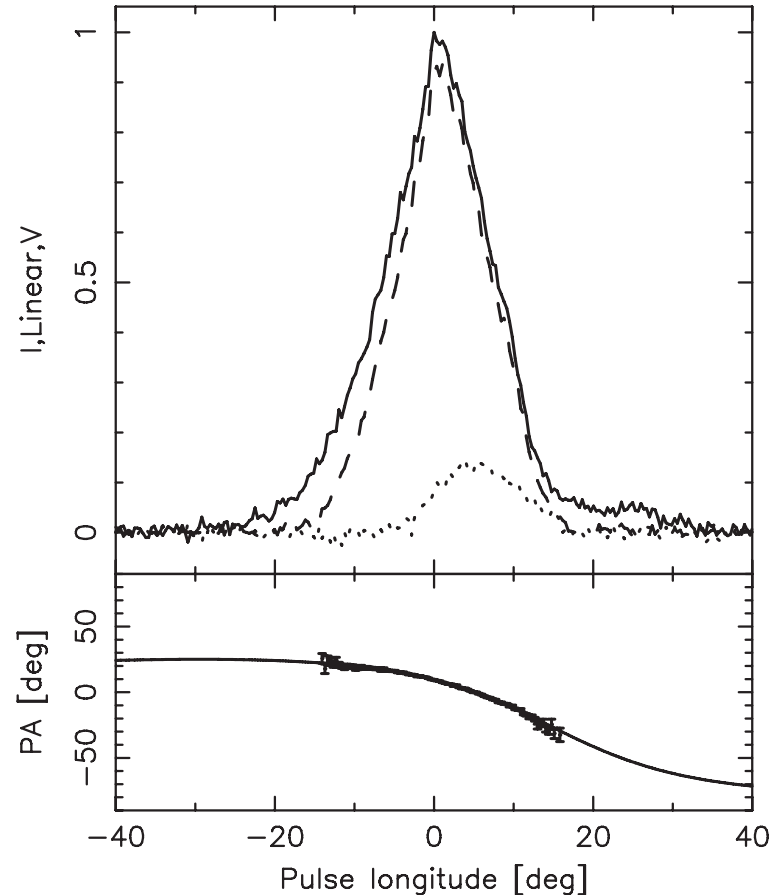

Figure 6. As in Figure 2, but for PSR J0659+1414 at $1418 \mathrm{MHz}$. These Arecibo data are taken from Weisberg et al. (1999).

ature distribution (i.e., hotter polar caps). At higher energies $(>1.5 \mathrm{keV})$, where the non-thermal component dominates, the pulsed fraction increases and the profile becomes single peaked.

A relatively aligned geometry is consistent with the sinusoidal soft X-ray profile, which suggests only one pole is visible from Earth and the low amount of modulation in soft X-rays also hints toward an aligned rotator (De Luca et al. 2005). However, there are some complications in modeling of the soft $\mathrm{X}$-rays, because there is an apparent anti-correlation between the hot and cool blackbody component of the thermal part of the emission that is not easily understood without invoking significant multipole components of the magnetic field or magnetospheric reprocessing of thermal photons. In addition, the best-fitting emitting radius of $\sim 21 \mathrm{~km}$ (using the very accurate radio VLBI parallax measurement) is unlikely given the expectations for a standard neutron star.

\subsubsection{Optical}

PSR J0659+1414 is seen at optical wavelengths (Caraveo et al. 1994), allowing the study of the (non-thermal) optical pulsations (e.g., Kern et al. 2003). The optical light curve is double peaked (see Figure 5) and is very similar to that seen in near-UV (Shibanov et al. 2005; Kargaltsev \& Pavlov 2007). The first optical peak following the radio pulse is aligned with the single $\gamma$-ray peak, while the second optical peak following the radio peak is aligned with the peak seen in (non-thermal) $\mathrm{X}$-rays above $1.5 \mathrm{keV}$.

\subsubsection{Radio}

The radio profile of PSR J0659+1414 is roughly triangular at $1.4 \mathrm{GHz}$ (see Figure 6) with a weak shoulder at the trailing edge. The weak shoulder is associated with weak and broad radio pulses, while the radio pulses in the triangle have a "spiky" appearance (Weltevrede et al. 2006b). The strongest of these radio pulses are argued (Weltevrede et al. 2006a) to be similar to transient radio bursts seen from the so-called rotating radio transients (RRATs; McLaughlin et al. 2006). The radio emission 


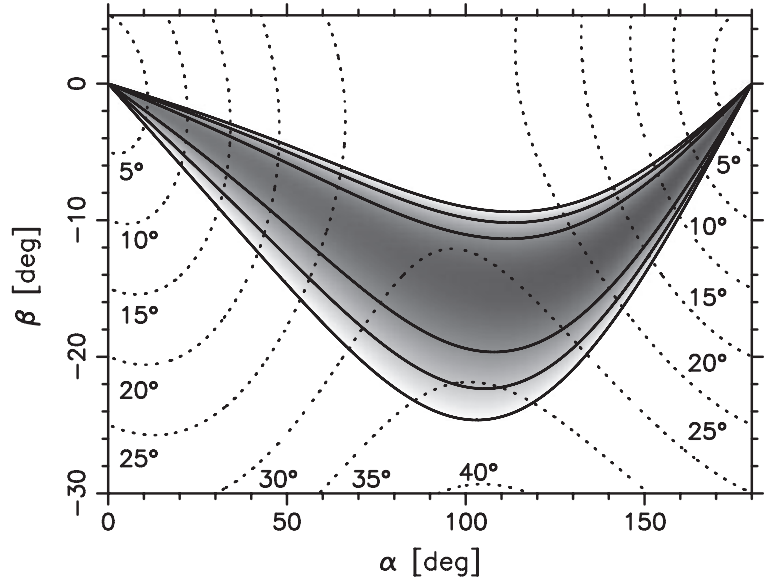

Figure 7. As in Figure 3, but for PSR J0659+1414. The lowest reduced $\chi^{2}$ is 0.93 .

is almost completely linearly polarized at $1.4 \mathrm{GHz}$, but there is significant depolarization at the leading edge. There is also significant circular polarization, which is strongest at the trailing edge of the profile. Curiously, at 6.2 and $8.4 \mathrm{GHz}$ the profile is completely depolarized (Johnston et al. 2006 and unpublished Parkes data ${ }^{68}$ ).

Figure 7 shows the $\chi^{2}$ map of the RVM fit for a high signal-to-noise radio profile taken from Weisberg et al. (1999). The results are consistent with previous results (e.g., Lyne \& Manchester 1988; Rankin 1993; Everett \& Weisberg 2001), and it is immediately clear that, as is often the case, $\alpha$ is unconstrained.

The steepest gradient of the P.A.-swing lags the peak of the radio peak by $14.9 \pm 0.7$ (Everett \& Weisberg 2001), suggesting an emission height of $1200 \mathrm{~km}$ (Equation (5)) and $\rho=22^{\circ}$ (using Equation (7)), implying that $\alpha$ should be $\sim 50^{\circ}$ (see Figure 7). The weak shoulder at pulse phases $18^{\circ}-30^{\circ}$ in Figure 6 could indicate that the peak of the profile leads the pulse phase corresponding to fiducial plane, which would suggest that $\alpha \lesssim 50^{\circ}$. A relatively aligned geometry would be in line with the above discussion about the thermal X-rays. The optical light curve is linearly polarized and optical P.A. points can be measured (Kern et al. 2003). Unfortunately, while the optical linear polarization is potentially useful, the limited data (three phase bins) and large errors make it impossible at present to use these data to constrain the RVM fit. Improved optical polarization measurements, however, have the potential to greatly refine our geometrical knowledge of this important pulsar.

\subsection{PSR J0742-2822 (B0740-28)}

\subsubsection{The Pulsar and its Surroundings}

PSR J0742-2822 was discovered as a radio pulsar by Facondi et al. (1973). Koribalski et al. (1995) determined a kinematic distance between $2.0 \pm 0.6$ and $6.9 \pm 0.8 \mathrm{kpc}$, which was higher than the distance derived from the DM according to the Taylor \& Cordes 1993 model $(1.9 \mathrm{kpc})$. This was argued by Koribalski et al. (1995) to be caused by an overestimation of the electron density of the Gum Nebula in this position. There is a steep gradient in the electron density in this direction in the Cordes \& Lazio (2002) model, which was constructed such that its predicted distance is consistent with the kinematic distance. We therefore adopt a distance of $2 \mathrm{kpc}$ with the note that it could possibly be as distant as $7 \mathrm{kpc}$.

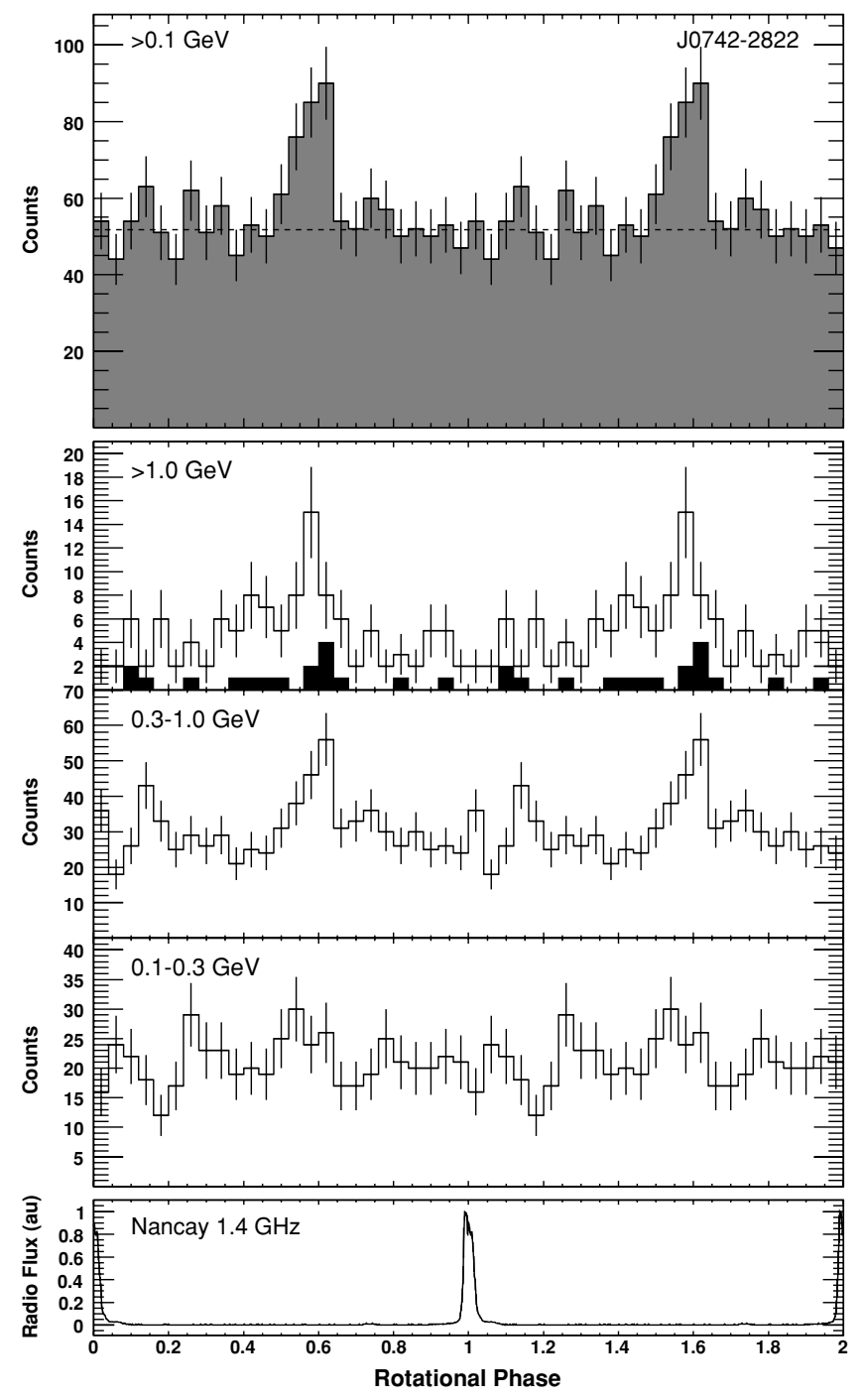

Figure 8. As in Figure 1, but for PSR J0742-2822.

$$
\text { 4.3.2. } \gamma \text {-rays }
$$

PSR J0742-2822 has a relatively narrow single peak in $\gamma$-rays, especially above $1 \mathrm{GeV}$ (see Figure 8 ). The $\gamma$-ray peak lags the radio peak by 0.61 in phase. In contrast to PSR $\mathrm{J} 0659+1414$, this pulsar is much weaker at low energies and is not detected below $300 \mathrm{MeV}$. Note that if the pulsar is at a distance of $7 \mathrm{kpc}$ (the upper limit of the kinematic distance), the $\gamma$-ray efficiency would be $\sim 70 \%$. This large efficiency may indicate that the pulsar is nearer than the distance upper limit.

\subsubsection{Radio}

Like the other pulsars discussed so far, the radio emission of this pulsar is highly linearly polarized, but there is depolarization at the trailing edge (see Figure 9). The circular polarization changes sign roughly in the middle of the profile, which is often associated with emission coming from close to the magnetic axis (Radhakrishnan \& Rankin 1990).

The $\chi^{2}$ map of the RVM fit (Figure 10) is much better constrained than for the other pulsars. The reduced $\chi^{2}$ of the best fit is not good (6.9), because the observed P.A.-swing deviates slightly from the model at the far trailing side of the profile (see Figure 9). This deviation is caused by a small jump in P.A. at the 


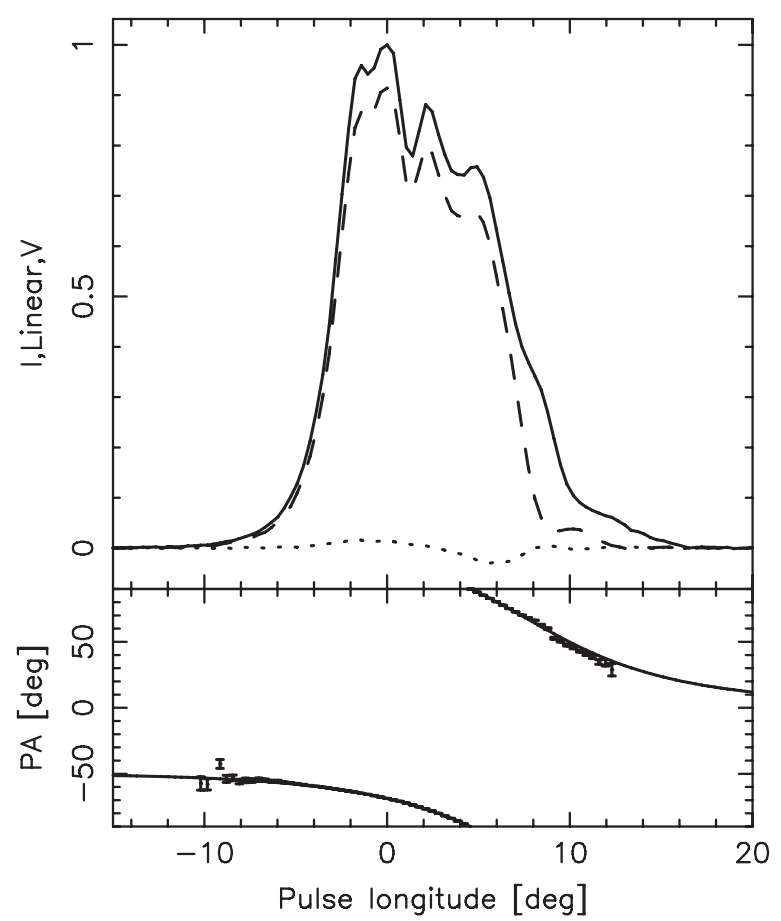

Figure 9. As in Figure 2, but for Parkes data of PSR J0742-2822 at 1369 MHz.

pulse phase corresponding to the pulse phase where significant depolarization is observed.

Weltevrede \& Johnston (2008) derived that $h_{\text {P.A. }}=350 \mathrm{~km}$, based on the observed offset between the center of the radio pulse profile and the location of the steepest gradient of the P.A.-swing (slightly larger than that derived by, e.g., von Hoensbroech \& Xilouris 1997), suggesting $\rho=18^{\circ}$ (Equation (7)). This opening angle is inconsistent with the RVM fit (see Figure 10), which suggests that the half opening angle of the radio beam $\rho \lesssim 15^{\circ}$ (in line with the value of $\rho$ derived by, e.g., Kramer et al. 1994). Possibly the beam is asymmetric with respect to the magnetic axis (e.g., Lyne \& Manchester 1988). Nevertheless, if we believe that most of the open field line region is active, this pulsar is unlikely to be aligned and we expect $\beta \sim-7^{\circ}$.

\subsection{PSR J1420-6048}

\subsubsection{The Pulsar and its Surroundings}

PSR J1420-6048 is a 68 ms pulsar (D'Amico et al. 2001) in the northeast wing of the complex of compact and extended radio sources known as Kookaburra (Roberts et al. 2001). It is located within the 0.32 wide $95 \%$ confidence level radius of the center of 3EG J1420-6038. The pulsar's large spin-down power and distance makes it a plausible match for the EGRET source. The situation is complicated by the Fermi LAT discovery of pulsations from an X-ray source in the Rabbit PWN, PSR J1418-6058 with $\dot{E}=5 \times 10^{36} \mathrm{erg} \mathrm{s}^{-1}$, only 0.24 away from the radio pulsar, and 0.54 from the EGRET source (Abdo et al. $2009 \mathrm{~b}$ ). The period is $110 \mathrm{~ms}$, different from the weak detection of pulsed X-rays with period $108 \mathrm{~ms}$ reported by $\mathrm{Ng}$ et al. (2005). There are also three $\mathrm{TeV}$ sources in this region discovered by HESS (Aharonian et al. 2006, 2008), but the association between the $\mathrm{TeV}$ sources and the $\gamma$-ray pulsars is unclear.

PSR J1420-6048 is the youngest and most energetic pulsar of our sample. The pulsar distance, derived from the DM to be $5.6 \pm 0.9 \mathrm{kpc}$ according to the Cordes \& Lazio (2002) model,

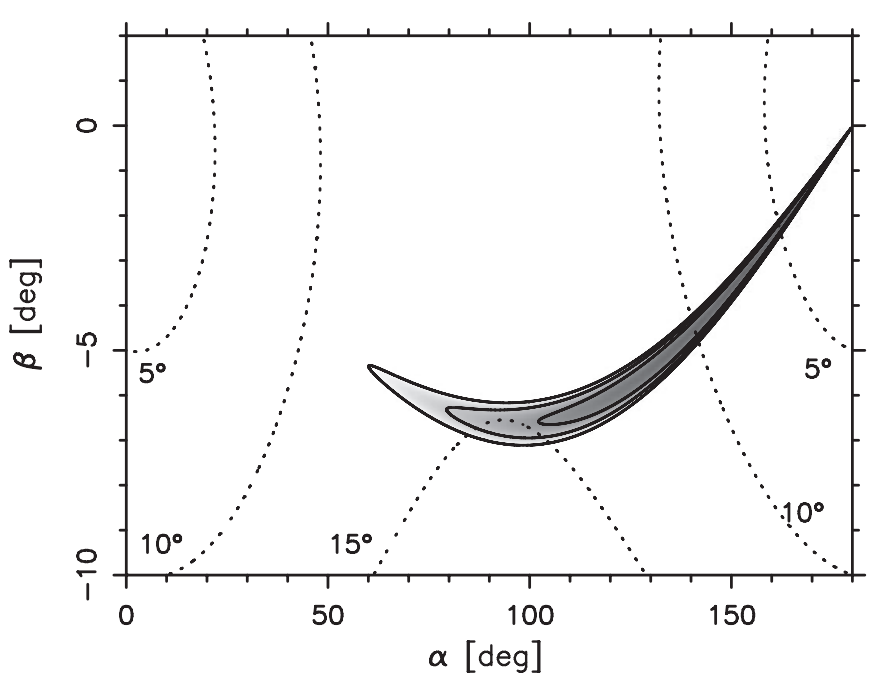

Figure 10. As in Figure 3, but for PSR J0742-2822. The lowest reduced $\chi^{2}$ is 6.9 .

is consistent with the X-ray absorption of $N_{\mathrm{H}} \sim 2 \times 10^{22} \mathrm{~cm}^{-2}$ (Roberts et al. 2001) but subject to uncertainties because of HII regions and dense clouds in the Carina arm. In this paper, we apply a more conservative distance uncertainty of $30 \%$ to our adopted DM distance estimates (so $5.6 \pm 1.9 \mathrm{kpc}$ for this pulsar). This is to try to take into account systematic errors in the model for the electron density in the Galaxy and this approach is identical to that in the LAT pulsar catalog paper (Abdo et al. 2009e).

$$
\text { 4.4.2. } \gamma \text {-rays }
$$

PSR J1420-6048 is the brightest $\gamma$-ray pulsar of our sample. The light curve of PSR J1420-6048 (see Figure 11) has a broad peak, which probably consists of two components lagging the second radio peak by 0.26 and 0.44 in phase. This would imply a peak separation $\Delta=0.18$. The second component may follow the common Fermi pulsar pattern of increasing dominance at high $\gamma$-ray energies (Abdo et al. 2009e). The small angular separation between this pulsar and J1418-6058 (Abdo et al. 2009b) considerably increases the background flux at the position of the pulsar over a large energy interval. The estimation of the background level, which follows from a simple measurement of the flux in a $1^{\circ}-2^{\circ}$ ring around the pulsar, does not take into account the flux of J1418-6058, and as a consequence the background level is underestimated. The $\gamma$-ray spectrum is fitted significantly better by including an exponential cutoff.

$$
\text { 4.4.3. X-rays }
$$

There is a marginal detection of $\mathrm{X}$-ray pulsations at the radio pulse period by ASCA (see Figure 12) from within the X-ray nebula AX J1420.1-6049 (Roberts et al. 2001). The X-ray and $\gamma$-ray light curves peak at different phases with respect to the radio peak, so it is not clear how the $\gamma$-rays and $\mathrm{X}$-rays are related.

\subsubsection{Radio}

PSR J1420-6048 is another example of a radio pulsar that is nearly completely linearly polarized (e.g., Johnston \& Weisberg 2006) and there is also some circular polarization (see Figure 13). The radio pulse profile shows a double-peaked 

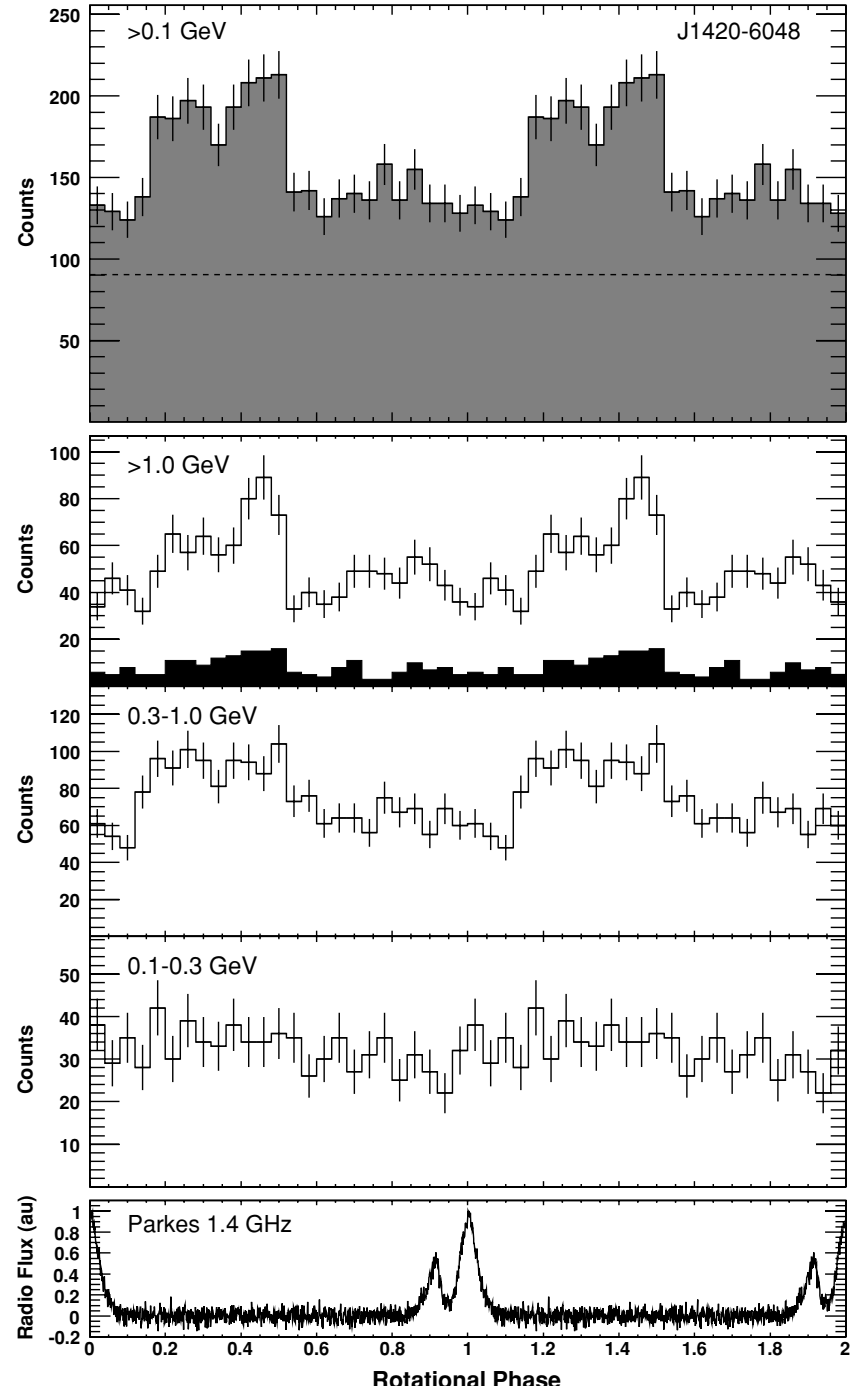

Figure 11. As in Figure 1, but for PSR J1420-6048.

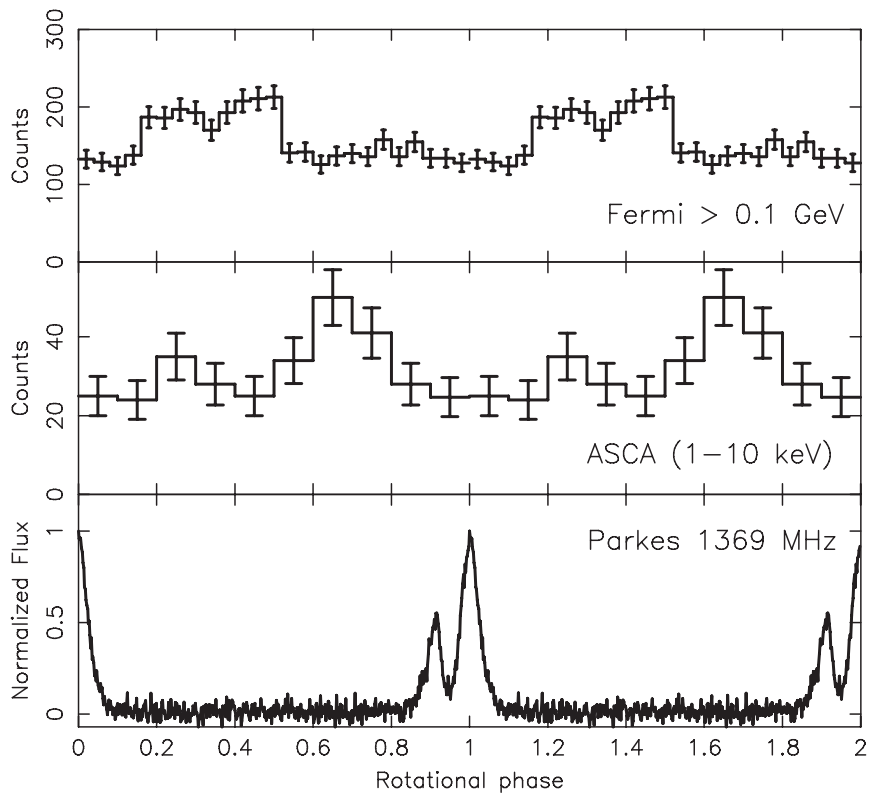

Figure 12. Compilation of phase-aligned light curves of PSR J1420-6048 as seen by Fermi, ASCA (Roberts et al. 2001), and Parkes. The X-ray light curve has an absolute phase error $\sim 0.06$.

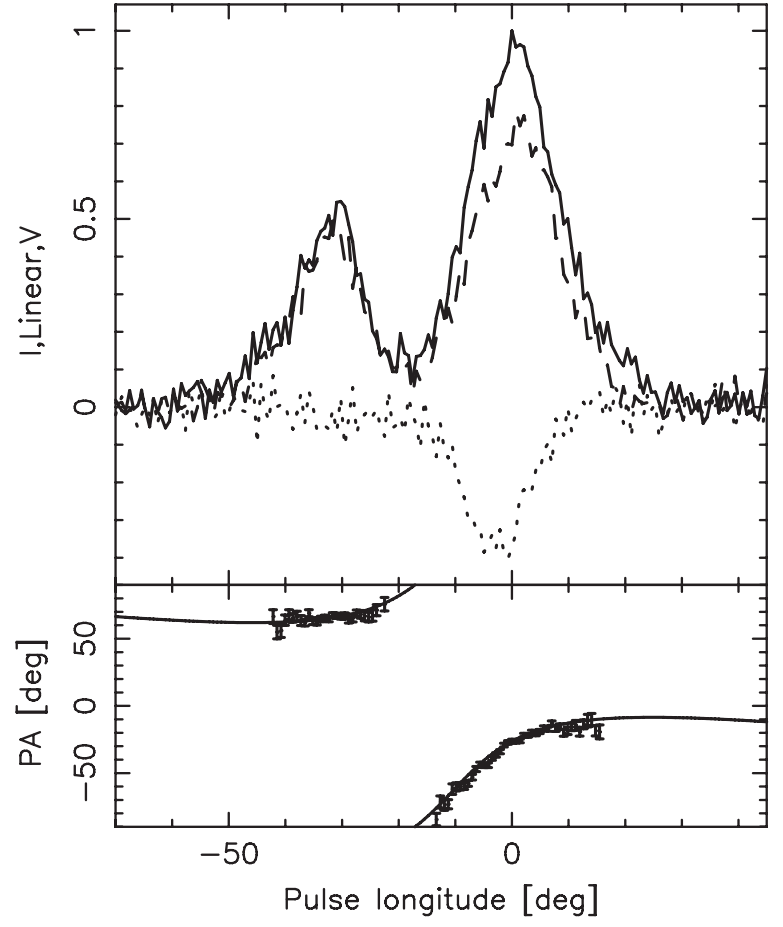

Figure 13. As in Figure 2, but for Parkes data of PSR J1420-6048 at 1369 MHz.

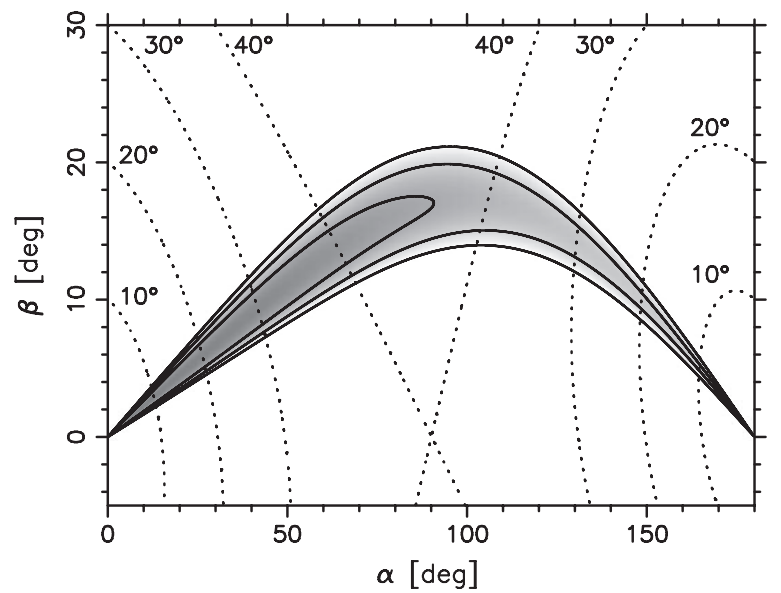

Figure 14. As in Figure 3, but for PSR J1420-6048. The lowest reduced $\chi^{2}$ is 1.3 .

structure with the trailing component being strongest, something that is generally seen for young pulsars with characteristic ages less than 75 kyr (Johnston \& Weisberg 2006).

RVM modeling of the P.A.-swing has been carried out by Roberts et al. (2001), who claim $\alpha \leqslant 35^{\circ}$ and $\beta \sim 0.5$. It is clear from Figure 14 that the best solution can be found at low $\alpha$ values, but neither $\alpha$ nor $\beta$ are well constrained. This geometry would be consistent with the arc of emission around the pulsar seen in the Chandra image ( $\mathrm{Ng}$ et al. 2005), which suggests (if interpreted as a torus) a $\zeta$ which is not particularly small or large.

Weltevrede \& Johnston (2008) found that $h_{\text {P.A. }}=100 \mathrm{~km}$, which is of the same order as what was found by (Johnston \& Weisberg 2006; $175 \mathrm{~km}$ ). Using the emission height of $100 \mathrm{~km}$ in Equation (7) leads to an expected $\rho$ of $15^{\circ}$. This implies (see Figure 14) that the magnetic inclination angle $\alpha$ should be relatively small $\left(\sim 20^{\circ}\right)$. As always, this derivation relies on the assumption that the pulsar beam is symmetric and nearly filled. 
PSR J1420-6048 belongs to a group of young pulsars with very wide profiles and relatively high values of $\dot{E} \gtrsim 5 \times$ $10^{35}$ erg s $^{-1}$ (e.g., Manchester 2005; Weltevrede \& Johnston 2008). They speculate that an analog can be drawn between the radio emission of these so-called "energetic wide beam pulsars" and their high-energy emission. They argued that the sites in the pulsar magnetosphere that produce the radio emission could be very similar to those of the high-energy emission, leading to the prediction that there should be strong similarity between the radio and $\gamma$-ray light curves. Although the $\gamma$-ray light curve may indeed be double peaked, the $\gamma$-ray light curve is significantly offset in phase from the radio profile, suggesting a significant difference in the location of production of the radio and $\gamma$-ray emission.

\subsection{PSR J1509-5850}

\subsubsection{The Pulsar and its Surroundings}

PSR J1509-5850 was discovered as a $89 \mathrm{~ms}$ radio pulsar by Kramer et al. (2003). It has a PWN as well as a long tail seen in X-rays (e.g., Kargaltsev et al. 2008) and radio (Hui \& Becker 2007). The distance to this pulsar estimated from the DM is $2.5 \pm 0.5 \mathrm{kpc}$ using the Cordes \& Lazio (2002) model, but, as discussed before, we adopt a more conservative distance uncertainty of $30 \%(2.5 \pm 0.8 \mathrm{kpc})$.

$$
\text { 4.5.2. } \gamma \text {-rays }
$$

This pulsar should not be confused with PSR B1509-58 (PSR J1513-5908), which was seen in soft $\gamma$-rays by BATSE, OSSE, and COMPTEL on CGRO (Ulmer et al. 1993). The light curve of PSR J1509-5850 (see Figure 15) is very broad. Similar to PSR J1420-6048, the light curve may be composed of two peaks lagging the radio profile by 0.18 and 0.39 in phase, respectively (corresponding to a peak separation $\Delta=0.21$ ). The spectrum of this pulsar shows a cutoff at $\sim 3 \mathrm{GeV}$ with the highest confidence of the pulsars discussed in this paper. The derived $\gamma$-ray efficiency is very high $(\sim 15 \%)$ and is the largest of the pulsars discussed in this paper (see Table 5). However, the distance to this pulsar is highly uncertain, therefore the luminosity and hence the $\gamma$-ray efficiency are not well constrained.

\subsubsection{Radio}

This pulsar is the weakest radio source of our sample. As noted by Weltevrede \& Johnston (2008), the correlation between $\dot{E}$ and a high degree of linear polarization does not hold for this pulsar (see Figure 16). The low degree of linear polarization in combination with a low overall $\mathrm{S} / \mathrm{N}$ prevents us from measuring the P.A.-swing, and therefore the emission geometry cannot be constrained for this pulsar using the RVM model.

\subsection{PSR J1718-3825}

\subsubsection{The Pulsar and its Surroundings}

PSR J1718-3825 is a 75 ms pulsar discovered by Manchester et al. (2001) at radio wavelengths. It has an associated X-ray nebula (Hinton et al. 2007) and an associated HESS source (Aharonian et al. 2007). Its distance derived from the DM is $3.6 \pm 0.4 \mathrm{kpc}$ according to the Cordes \& Lazio (2002) model, or $3.6 \pm 1.1 \mathrm{kpc}$ by applying a more conservative distance uncertainty of $30 \%$.
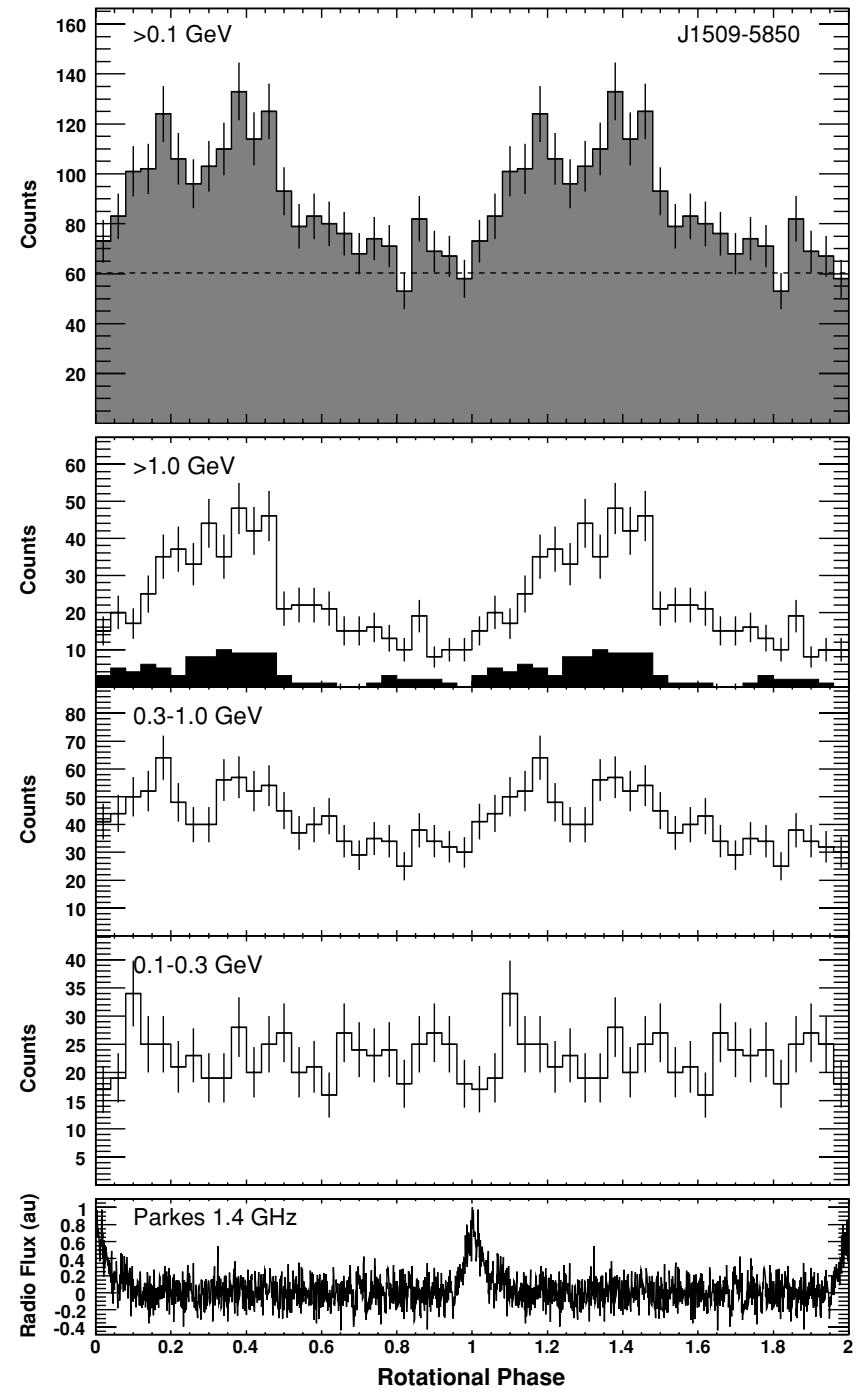

Figure 15. As in Figure 1, but for PSR J1509-5850.

$$
\text { 4.6.2. } \gamma \text {-rays }
$$

The light curve of PSR J1718-3825 is single peaked (FWHM is 0.20 in phase) and it lags the radio profile by 0.42 in phase (see Figure 17). Like the previous two pulsars discussed, its spectrum is significantly better described by including a cutoff energy. However, the cutoff energy itself cannot be determined well.

\subsubsection{Radio}

The radio pulse profile of J1718-3825 has a relatively complex shape, and it is highly linearly polarized (see Figure 18). There is some negative circular polarization as well. The $1.4 \mathrm{GHz}$ profile is very similar to what is seen at higher frequencies (unpublished Parkes data ${ }^{68}$ ).

The P.A.-swing is S-shaped, allowing an RVM fit, although it is not very constraining (see Figure 19). The steepest gradient of the RVM model lags the center of the radio pulse by $5^{\circ}$, corresponding to $h_{\text {P.A. }} \sim 80 \mathrm{~km}$ (Equation (5)) and an expected $\rho \sim 13^{\circ}$ (Equation (7)). This would imply that $\alpha \sim 20^{\circ}$ (see Figure 19), however, the complex shape of the radio profile makes it difficult to objectively determine which pulse phase corresponds to emission from the fiducial plane. If the peak of the profile is a better indicator for the magnetic axis than the center, then $\alpha$ is larger $\left(\sim 50^{\circ}\right)$. 


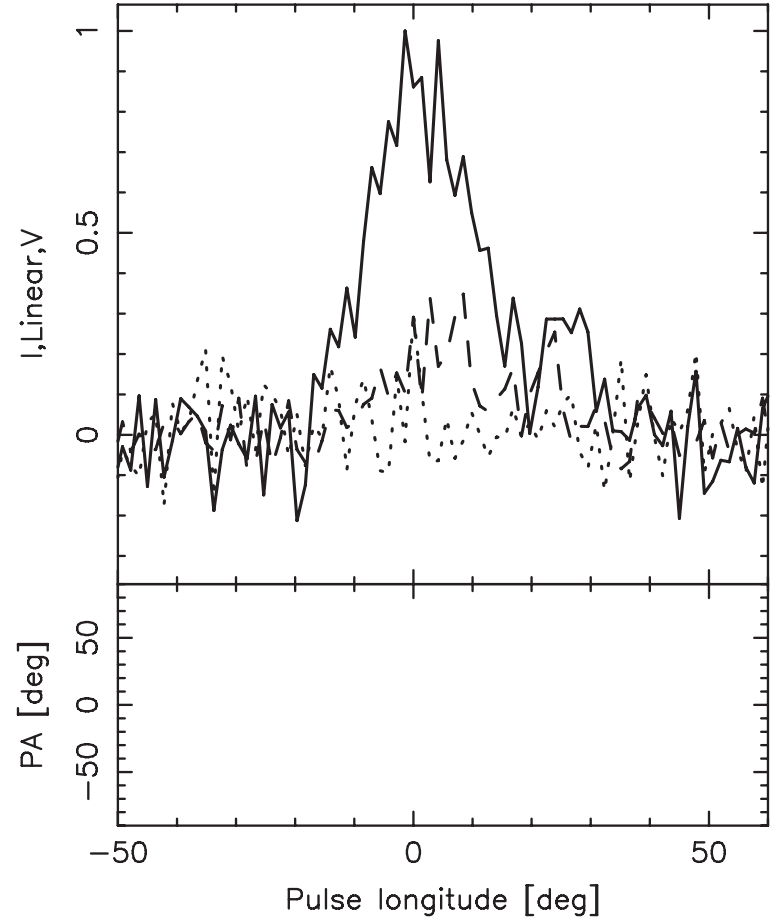

Figure 16. As in Figure 2, but for Parkes data of PSR J1509-5850 at 1369 MHz.

\section{DISCUSSION}

From examination of the light curves in the LAT pulsar catalog (Abdo et al. 2009e), certain groupings of $\gamma$-ray light curves are apparent. One group, of which the Vela pulsar is the archetypical example (Abdo et al. 2009c), is characterized by light curves which consist of two narrow peaks separated by $\sim 0.4-0.5$ in phase with the first peak offset from the radio profile by $\sim 0.1-0.2$ in phase. A second group of light curves also shows double peaks, but the peaks are much closer together in phase and can blend together to produce a more squarelooking light curve. Examples of this sort include PSR J17094429, the radio-quiet PSR J0007+7303 (Abdo et al. 2009b) and the radio-loud PSR J1057-5226 (Abdo et al. 2009e). The final group of light curves appears to consist of a single component, possibly because the peaks are completely blended together, are unresolved given the present $\mathrm{S} / \mathrm{N}$ or simply because they are truly single. There is some evidence that the closer doubles and single component light curves are found preferentially among the pulsar with lower $\dot{E}$, whereas the Vela-like light curves are found for all spin-down luminosities (Abdo et al. 2009e).

The light curves of the six pulsars discussed in this paper are all consistent with single peaks. Nevertheless, those of PSRs J1420-6048 and J1509-5850 could have the appearance of closely spaced doubles, therefore resembling that of PSR J1709-4429. The light curves of the other four pulsars do not show, at least given the current count statistics, any hint of a second pulse component. PSRs J0631+1036 and J1718-3825 have relatively broad pulses with an offset to the radio pulse of 0.4 in phase. The $\gamma$-ray emission of PSR J0631+1036 has a tail to earlier phase, visible especially above $1 \mathrm{GeV}$. The light curve of PSR J0742-2822 is narrower, and is offset from the radio peak by 0.61 in phase. Finally, PSR J0659+1414 has a single broad peak at the same phase as a corresponding peak in the optical and UV and yet lacks the second peak seen at those energies.

We now consider if outer magnetospheric models can explain these light curves in conjunction with the constraints on the

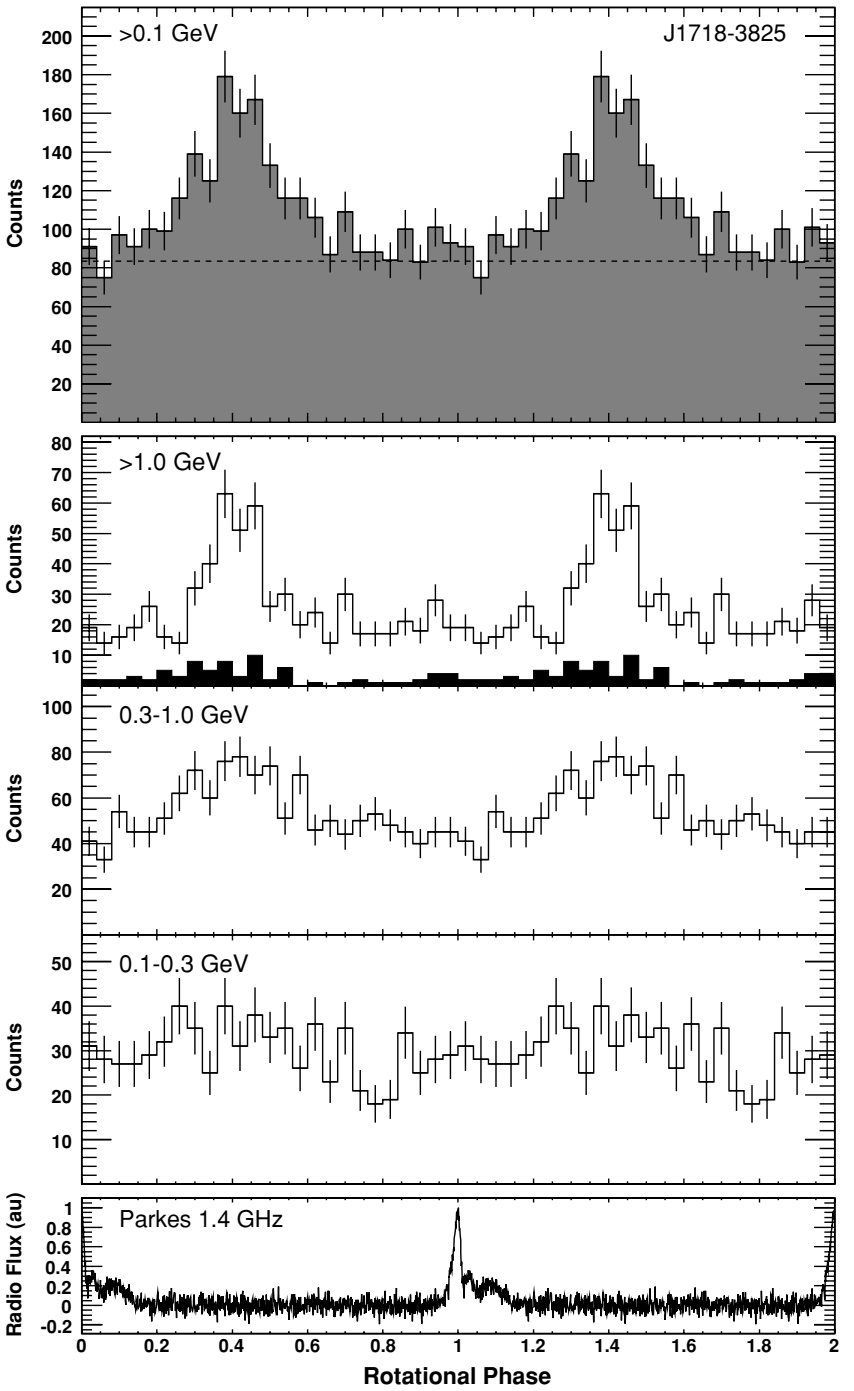

Figure 17. As in Figure 1, but for PSR J1718-3825.

geometry derived from the radio profiles by comparing the observed $\gamma$-ray light curves to those predicted in the two-pole caustic and outer-gap models of Watters et al. (2009).

In the context of the Watters et al. (2009) models, the light curves of PSRs J0631+1036, J1420-6048, J1509-5850, and J1718-3825 can all be described in term of closely spaced double peaks. For PSRs J1420-6048 and J1509-5850, there is some evidence for a double-peaked nature, while for PSRs J0631+1036 and J1718-3825 it is well possible that the peaks are unresolved due to a lack of S/N. Double-peaked light curves with small separations occur naturally at the correct phases relative to the radio emission in the outer-gap and two-pole caustic pictures, if one skims the edge of the outer magnetosphere cone. For narrow gaps (low-efficiency pulsars), this occurs for a range $\alpha \sim 40^{\circ}-50^{\circ}$ and $\zeta \sim 50^{\circ}-65^{\circ}$ for the outer-gap model and $\alpha \sim 45^{\circ}-60^{\circ}$ and $\zeta \sim 35^{\circ}-55^{\circ}$ in the twopole caustic model. For wider gaps (high-efficiency pulsars), the outer-gap model has many similar solutions extending to $\alpha, \zeta \sim 80^{\circ}$, but such pulses are more difficult to realize in the two-pole caustic geometry.

Single peaks that lag the radio pulse by $0.4-0.5$ in phase occur in both outer-gap and two-pole caustic models for the smallest gap widths, over a band spanning $\alpha \approx 55^{\circ}-75^{\circ}, \zeta \approx 20^{\circ}-50^{\circ}$. These are effectively the second peak of the more typical double 


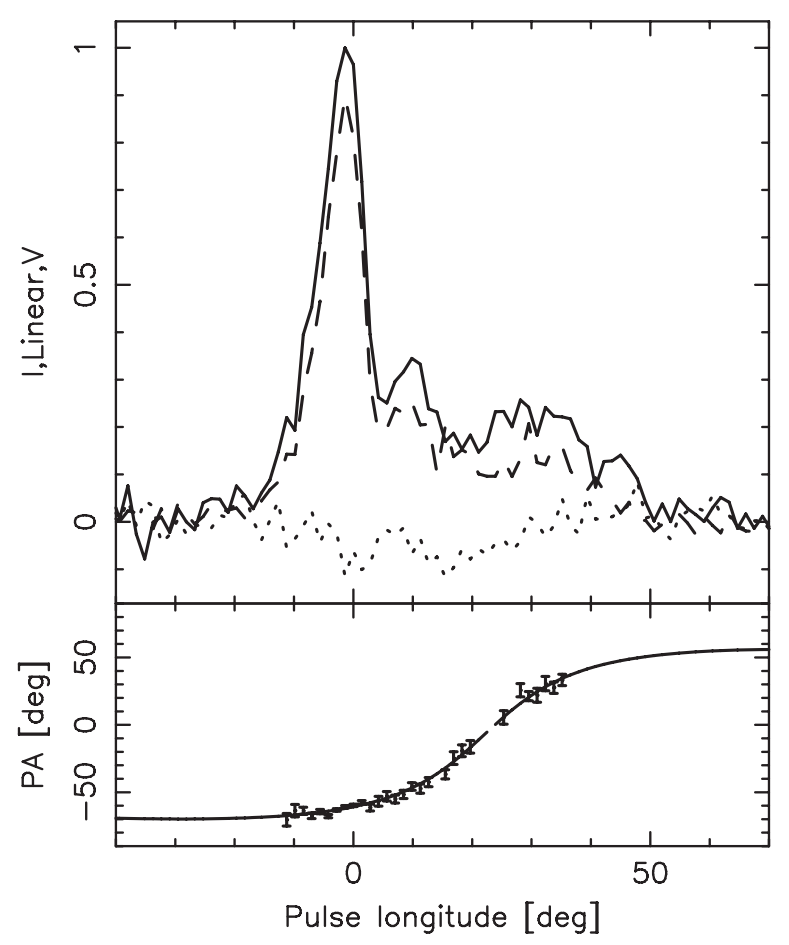

Figure 18. As in Figure 2, but for Parkes data of PSR J1718-3825 at $1369 \mathrm{MHz}$.

profile, with the first peak weak or absent in this angle range. The preferred geometry derived from the radio data of PSR J1420-6048 ( $\alpha \sim 20^{\circ}$ and $\beta \sim 5^{\circ}$ ) would favor the two-pole caustic model (the outer-gap model does not predict significant $\gamma$-ray pulsations for such a geometry). However, in this case we would expect the $\gamma$-ray peak to appear at phase $0.1-0.2$. In any case, at present the constraints derived from the radio data are too weak to draw firm conclusions.

If the light curve of PSR J0742-2822 consists only of a single narrow peak at phase 0.61 , it cannot easily be explained by either the outer-gap or the two-pole caustic model. However, it is tempting to associate this peak with the second peak seen in the Vela-like light curves as it has the correct phase offset with respect to the radio profile. If we do this then the first peak must either be missing entirely or at least be much weaker than the second peak; we note that the weak excess at phase 0.12 in Figure 8 is at the expected phase-longer integrations should eventually settle this. This picture would tie in nicely with the general trend of a relatively strong second peak at higher energies as seen for double-peaked light curves.

Finally, we discuss the curious case of PSR J0659+1414. It has a high $\mathrm{S} / \mathrm{N}$ light curve and is clearly single. The spin parameters of PSR J0659+1414 are not exceptional. Its $\dot{E}$ is relatively low, but that of PSR J1057-5226, which has a harder spectrum, is lower. However, the magnetic field strength at the light cylinder is very low for J0659+1414. A special geometry may be responsible for the unusual properties of this pulsar, and we consider two possible interpretations. In the first, PSR J0659+1414 is more Vela-like. In this scenario, the second component (visible at lower energies) is entirely missing at $\gamma$-ray energies. However, although the phase of the visible component can then be well explained by both outer-gap and two-pole caustic models, it implies relatively large values for $\alpha$ and $\zeta$, which is somewhat at odds with the constraints from the modeling of the radio data and the thermal X-rays. In the second interpretation, PSR J0659+1414 is an aligned rotator

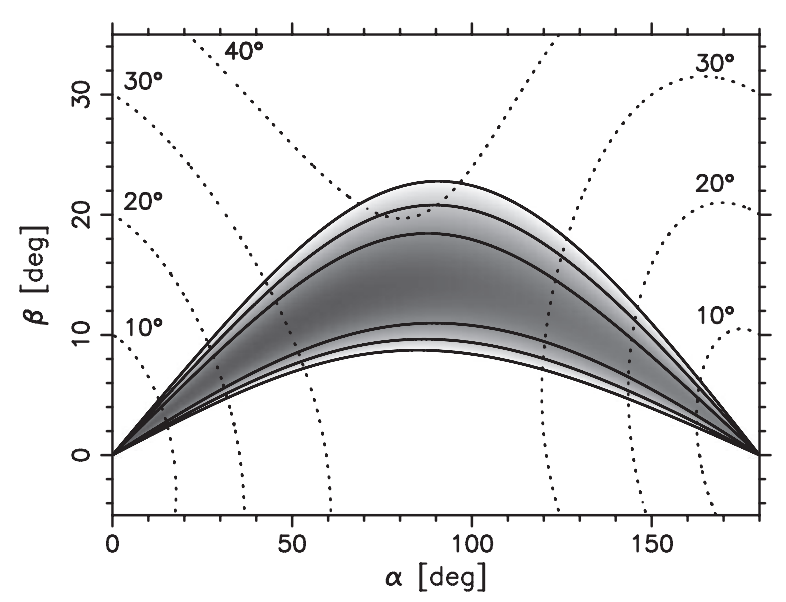

Figure 19. As in Figure 3 , but for PSR J1718-3825. The lowest reduced $\chi^{2}$ is 1.3 .

$\left(\alpha \lesssim 40^{\circ}\right)$. In this case, in the outer-gap picture one would not expect strong emission, but the two-pole caustic model does indeed show a single pulse at phase $0.1-0.2$ later than the radio emission. We also note that the pulsar has an extremely low efficiency (less than $\sim 1 \%$ ) and as its distance is well known and the flux correction factor $f_{\Omega}$ is likely to be of order unity there is little way to avoid this conclusion. Further, its spectrum is among the softest of all the pulsars detected by Fermi (Abdo et al. 2009e), and it is virtually undetected at energies $>1 \mathrm{GeV}$. This might also point to a special, aligned geometry where $\gamma$-ray emission at somewhat lower altitude $\left(\gtrsim 0.1 R_{\mathrm{LC}}\right)$ is being observed.

In summary, therefore, we find that both the two-pole caustic model and the outer-gap model do a good job in predicting the phase offset with respect to the radio emission for PSRs J0631+1036, J1420-6048, J1509-5850, and J1718-3825 under the assumption that these are closely spaced double-peaked $\gamma$-ray emitters. We note that the light curve shape, especially of PSR J1718-3825, is not well predicted by the models. However, it should be stressed that the model calculations in Watters et al. (2009) are idealized and therefore one would indeed only expect approximate agreement between the models and the data. One could speculate that to obtain more realistic physical models one should include a larger range of field lines from which $\gamma$-ray photons are produced. This smooths out the sharp peaks in the light curves caused by caustics; these sharp peaks are not observed for the pulsars in this paper. This also has the effect of making the so-called "bridge" of emission between the peaks stronger, causing the peaks in the light curve to blended together. In Watters et al. (2009), the two-pole caustic and outer-gap widths increase with $\dot{E}$, but the emission region is an infinitely thin strip on the gap inner edge. For PSR J07422822 , we surmise that there is a "missing" $\gamma$-ray peak at phase offset 0.1 (and possibly the second peak is "missing" for PSR J0659+1414). Without speculating about a missing peak, it is hard to understand the light curve of PSR J0659+1414 in the context of outer-gap models. It may be a roughly aligned rotator with $\gamma$-ray emission from lower down in the magnetosphere.

\section{CONCLUSIONS}

We report here on the detection of pulsed $\gamma$-rays by Fermi for PSRs J0631+1036, J0659+1414, J0742-2822, J1420-6048, $\mathrm{J} 1509-5850$, and $\mathrm{J} 1718-3825$. These six pulsars are young to middle-aged and, except for PSR J1420-6048, have relatively 
small values of $\dot{E}$ compared to other known $\gamma$-ray pulsars. In all cases, the $\gamma$-ray light curves appear single peaked (at least with the present count statistics), but there is a hint that at least two of them have closely spaced double peaks. As Fermi continues its all-sky survey, the quality of the light curves will increase, helping to resolve this issue.

We present high-quality radio polarization profiles for these pulsars and discuss their geometries in the context of RVM fitting and simple beam modeling. Unfortunately, the narrow phase range of the radio emission generally leaves a strong degeneracy between the fit $\alpha$ and $\beta$ values. This, in combination with the limited $\gamma$-ray count statistics makes it difficult to distinguish between single-peaked and double-peaked light curves and hence to make the comparison with the model predictions. We show that models where the $\gamma$-ray emission occurs at relatively high altitudes in the pulsar magnetosphere, such as the outer-gap or two-pole caustic models, do a good job in predicting the phase of the $\gamma$-ray emission relative to the radio emission. However, the shape of the $\gamma$-ray light curve is less well modeled, and additional inputs to the model are needed to explain the strong bridge emission in some pulsars and the strong single $\gamma$-ray component in PSR J0742-2822 in particular. Finally, PSR J0659+1414 warrants further attention. It has a peculiar light curve and phase offset with the radio profile, its $\gamma$-ray efficiency is low, and its $\gamma$-ray spectrum is extremely soft. It may be an aligned rotator with $\gamma$-ray emission arising relatively low down in the magnetosphere. Higher $\mathrm{S} / \mathrm{N} \gamma$-ray light curves in combination with possible additional geometrical constraints, such as from PWN imaging, will result in stronger constraints on the models.

The Australia Telescope is funded by the Commonwealth of Australia for operation as a National Facility managed by the CSIRO. The Nançay Radio Observatory is operated by the Paris Observatory, associated with the French Centre National de la Recherche Scientifique (CNRS). The Lovell Telescope is owned and operated by the University of Manchester as part of the Jodrell Bank Centre for Astrophysics with support from the Science and Technology Facilities Council of the United Kingdom. The Fermi LAT Collaboration acknowledges generous ongoing support from a number of agencies and institutes that have supported both the development and the operation of the LAT as well as scientific data analysis. These include the National Aeronautics and Space Administration and the Department of Energy in the United States, the Commissariat à l'Energie Atomique and the Centre National de la Recherche Scientifique/Institut National de Physique Nucléaire et de Physique des Particules in France, the Agenzia Spaziale Italiana and the Istituto Nazionale di Fisica Nucleare in Italy, the Ministry of Education, Culture, Sports, Science and Technology (MEXT), High Energy Accelerator Research Organization (KEK) and Japan Aerospace Exploration Agency (JAXA) in Japan, and the K. A. Wallenberg Foundation, the Swedish Research Council and the Swedish National Space Board in Sweden. Additional support for science analysis during the operations phase is gratefully acknowledged from the Istituto Nazionale di Astrofisica in Italy and the Centre National d'Études Spatiales in France.

\section{REFERENCES}

Abdo, A. A., et al. 2009a, ApJ, submitted (Crab paper) (arXiv:0911.2412) Abdo, A. A., et al. 2009b, Science, 325, 840 (blind search paper)
Abdo, A. A., et al. 2009c, ApJ, 696, 1084 (Vela paper)

Abdo, A. A., et al. 2009d, ApJS, 183, 46 (bright source list paper)

Abdo, A. A., et al. 2009e, ApJ, submitted, arXiv:0910.1608 (pulsar catalogue paper)

Aharonian, F., et al. 2006, A\&A, 456, 245

Aharonian, F., et al. 2007, A\&A, 472, 489

Aharonian, F., et al. 2008, A\&A, 477, 353

Atwood, W. B., et al. 2009, ApJ, 697, 1071

Becker, W., \& Trümper, J. 1997, A\&A, 326, 682

Blaskiewicz, M., Cordes, J. M., \& Wasserman, I. 1991, ApJ, 370, 643

Brisken, W. F., Thorsett, S. E., Golden, A., \& Goss, W. M. 2003, ApJ, 593, L89

Caraveo, P. A., Bignami, G. F., \& Mereghetti, S. 1994, ApJ, 422, L87

Cheng, K. S., Ho, C., \& Ruderman, M. 1986, ApJ, 300, 500

Cognard, I., Theureau, G., Desvignes, G., \& Ferdman, R. 2009, Proc. Windows on the Universe, XXI Rencontres de Blois, arXiv:0911.1612

Cordes, J. M., \& Lazio, T. J. W. 2002, arXiv:astro-ph/0207156

Córdova, F. A., Hjellming, R. M., Mason, K. O., \& Middleditch, J. 1989, ApJ, 345,451

Crawford, F., Manchester, R. N., \& Kaspi, V. M. 2001, AJ, 122, 2001

D'Amico, N., et al. 2001, ApJ, 552, L45

Daugherty, J. K., \& Harding, A. K. 1996, ApJ, 458, 278

de Jager, O. C., Raubenheimer, B. C., \& Swanepoel, J. W. H. 1989, A\&A, 221, 180

De Luca, A., Caraveo, P. A., Mereghetti, S., Negroni, M., \& Bignami, G. F. 2005, ApJ, 623, 1051

Dyks, J. 2008, MNRAS, 391, 859

Dyks, J., \& Harding, A. K. 2004, ApJ, 614, 869

Dyks, J., \& Rudak, B. 2003, ApJ, 598, 1201

Dyks, J., Rudak, B., \& Harding, A. K. 2004, ApJ, 607, 939

Edwards, R. T., Hobbs, G. B., \& Manchester, R. N. 2006, MNRAS, 372, 1549

Everett, J. E., \& Weisberg, J. M. 2001, ApJ, 553, 341

Facondi, S. R., Salter, C. J., \& Sutton, J. M. 1973, A\&A, 27, 67

Gaensler, B. M., Stappers, B. W., Frail, D. A., Moffett, D. A., Johnston, S., \& Chatterjee, S. 2000, MNRAS, 318, 58

Gil, J. A., Gronkowski, P., \& Rudnicki, W. 1984, A\&A, 132, 312

Han, J. L., \& Manchester, R. N. 2001, MNRAS, 320, L35

Harding, A. K., Grenier, I. A., \& Gonthier, P. L. 2007, Ap\&SS, 309, 221

Hinton, J. A., Funk, S., Carrigan, S., Gallant, Y. A., de Jager, O. C., Kosack, K., Lemière, A., \& Pühlhofer, G. 2007, A\&A, 476, L25

Hobbs, G. B., Edwards, R. T., \& Manchester, R. N. 2006, MNRAS, 369, 655

Hobbs, G., Lyne, A. G., Kramer, M., Martin, C. E., \& Jordan, C. 2004, MNRAS, 353, 1311

Hui, C. Y., \& Becker, W. 2007, A\&A, 470, 965

Johnston, S., Karastergiou, A., \& Willett, K. 2006, MNRAS, 369, 1916

Johnston, S., \& Weisberg, J. M. 2006, MNRAS, 368, 1856

Kargaltsev, O., Misanovic, Z., Pavlov, G. G., Wong, J. A., \& Garmire, G. P. 2008, ApJ, 684, 542

Kargaltsev, O., \& Pavlov, G. G. 2007, Astrophys. Space Science, 308, 287

Keith, M. J., Johnston, S., Weltevrede, P., \& Kramer, M. 2009, MNRAS, in press (arXiv:0910.4778)

Kennea, J., Cordova, F., Chatterjee, S., Cordes, J., Ho, C., Much, R., Oosterbroek, T., \& Parmar, A. 2002, Proc. Symp. New Visions of the X-ray Universe in the XMM-Newton and Chandra Era, arXiv:astro-ph/0202055

Kern, B., Martin, C., Mazin, B., \& Halpern, J. P. 2003, ApJ, 597, 1049

Koribalski, B., Johnston, S., Weisberg, J. M., \& Wilson, W. 1995, ApJ, 441, 756

Kramer, M., Wielebinski, R., Jessner, A., Gil, J. A., \& Seiradakis, J. H. 1994, A\&AS, 107, 515

Kramer, M., et al. 2003, MNRAS, 342, 1299

Lorimer, D. R., \& Kramer, M. 2005, Handbook of Pulsar Astronomy (Cambridge: Cambridge Univ. Press)

Lyne, A. G., \& Manchester, R. N. 1988, MNRAS, 234, 477

Manchester, R. N. 2005, Ap\&SS, 297, 101

Manchester, R. N., Lyne, A. G., Taylor, J. H., Durdin, J. M., Large, M. I., \& Little, A. G. 1978, MNRAS, 185, 409

Manchester, R. N., et al. 2001, MNRAS, 328, 17

Marshall, H. L., \& Schulz, N. S. 2002, ApJ, 574, 377

Mattox, J. R., et al. 1996, ApJ, 461, 396

McLaughlin, M. A., et al. 2006, Nature, 439, 817

Morini, M. 1983, MNRAS, 202, 495

Muslimov, A. G., \& Harding, A. K. 2004, ApJ, 606, 1143

Ng, C.-Y., Roberts, M. S. E., \& Romani, R. W. 2005, ApJ, 627, 904

Ng, C.-Y., \& Romani, R. W. 2008, ApJ, 673, 411

Petrova, S. A. 2006, MNRAS, 368, 1764

Qiao, G. J., Manchester, R. N., Lyne, A. G., \& Gould, D. M. 1995, MNRAS, 274,572 
Radhakrishnan, V., \& Cooke, D. J. 1969, Astrophys. Lett., 3, 225

Radhakrishnan, V., \& Rankin, J. M. 1990, ApJ, 352, 258

Ramanamurthy, P. V., Fichtel, C. E., Kniffen, D. A., Sreekumar, P., \& Thompson, D. J. 1996, ApJ, 458, 755

Rankin, J. M. 1983, ApJ, 274, 333

Rankin, J. M. 1993, ApJS, 85, 145

Roberts, M. S. E., Romani, R. W., \& Johnston, S. 2001, ApJ, 561, L187

Romani, R. W., \& Yadigaroglu, I.-A. 1995, ApJ, 438, 314

Shibanov, Y. A., Sollerman, J., Lundqvist, P., Gull, T., \& Lindler, D. 2005, A\&A, 440,693

Shibanov, Y. A., et al. 2006, A\&A, 448, 313

Smith, D. A., et al. 2008, A\&A, 492, 923

Standish, E. M. 1998, JPL Planetary and Lunar Ephemerides, DE405/ LE405, Memo IOM 312.F-98-048 (Pasadena: JPL), http://ssd.jpl.nasa.gov/ iau-comm4/de405iom/de405iom.pdf

Strong, A. W., Moskalenko, I. V., \& Reimer, O. 2004, ApJ, 613, 962

Taylor, J. H., \& Cordes, J. M. 1993, ApJ, 411, 674
Thorsett, S. E., Benjamin, R. A., Brisken, W. F., Golden, A., \& Goss, W. M. 2003, ApJ, 592, L71

Torii, K., et al. 2001, ApJ, 551, L151

Ulmer, M. P., et al. 1993, ApJ, 417, 738

von Hoensbroech, A., Lesch, H., \& Kunzl, T. 1998, A\&A, 336, 209

von Hoensbroech, A., \& Xilouris, K. M. 1997, A\&AS, 126, 121

Watters, K. P., Romani, R. W., Weltevrede, P., \& Johnston, S. 2009, ApJ, 695, 1289

Weisberg, J. M., et al. 1999, ApJS, 121, 171

Weltevrede, P., \& Johnston, S. 2008, MNRAS, 391, 1210

Weltevrede, P., Stappers, B. W., Rankin, J. M., \& Wright, G. A. E. 2006a, ApJ, 645, L149

Weltevrede, P., \& Wright, G. 2009, MNRAS, 395, 2117

Weltevrede, P., Wright, G. A. E., Stappers, B. W., \& Rankin, J. M. 2006b, A\&A, 458,269

Weltevrede, P., et al. 2009, PASA, in press (arXiv:0909.5510)

Zepka, A., Cordes, J. M., Wasserman, I., \& Lundgren, S. C. 1996, ApJ, 456, 305 